\title{
Multi-attribute group decision-making for online education live platform selection based on linguistic intuitionistic cubic fuzzy aggregation operators
}

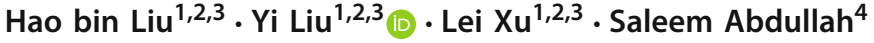 \\ Received: 22 June 2020 / Revised: 24 November 2020 / Accepted: 21 December 2020 \\ (c) SBMAC - Sociedade Brasileira de Matemática Aplicada e Computacional 2021
}

\begin{abstract}
The purpose of this study is to propose a multi-attribute group decision-making (MAGDM) method for online education live platform selection based on proposed novel aggregation operators (AOs) under linguistic intuitionistic cubic fuzzy set (LICFS). First, the Archimedean copula and co-copula are extended to handle linguistic intuitionistic cubic fuzzy information (LICFI) and the operational law of linguistic intuitionistic cubic fuzzy variables (LICFVs) based on extended copula (EC) and extended co-copula (ECC) are given. In addition, linguistic intuitionistic cubic fuzzy copula weighted average (LICFCWA) operator and linguistic intuitionistic cubic fuzzy copula weighted geometric (LICFCWG) operator are proposed based on EC and ECC under LICFI; meanwhile, some special forms of LICFCWA and LICFCWG have been obtained by different types generators of ECs and ECCs. Third, a novel MAGDM approach based on proposed LICFCWA (LICFCWG) is constructed to solve the selection problem of the online education live platform in the period of the COVID19 , and a detailed parameter analysis was carried out. Fourthly, LICFS will degenerate into linguistic intuitionistic fuzzy set and intuitionistic cubic fuzzy set, respectively, in different cases. Finally, some comparisons are carried out with other existing proposed MAGDM approaches. By comparing different types of experiments, the effectiveness and flexibility of the proposed approach are also showed.
\end{abstract}

Keywords Linguistic intuitionistic cubic fuzzy set - Extended copula · Extended co-copula $\cdot$ Aggregation operator $\cdot$ Multi-attribute group decision-making

Mathematics Subject Classification 90C29 · 91 B06

Communicated by Leonardo Tomazeli Duarte.

$\bowtie$ Yi Liu

liuyiyl@126.com

Extended author information available on the last page of the article

Published online: 08 January 2021

量 springer SD/MC 


\section{Introduction}

To solve the uncertain information in practical decision-making problems (DMPs), Zadeh (1965) proposed the fuzzy set (FS) in 1965. Then to improve the performance of FS, Atanassov (1986) proposed intuitionistic fuzzy set (IFS) by adding a non-membership degree (NMD). In the real DMPs, the situation may be more complex. Therefore, to solve these complex DMPs, and to make full use of the original information (in the decision-making process, the lost original information should be as few as possible), scholars have successively proposed interval-valued intuitionistic fuzzy set (IVIFS) (Atanassov and Gargov 1989), hesitation fuzzy set (HFS) (Torra 2010; Bedregal et al. 2014), dual hesitation fuzzy set (DHFS) (Zhu et al. 2012; Singh 2015), probability hesitation fuzzy set (PHFS) (Wang et al. 2014), probability interval-valued hesitation fuzzy set (PIVHFS) (Rodriguez et al. 2012), other forms (Chen et al. 2019; Khan 2019). The researches and applications of similar AOs have also become a research hotspot (Liu et al. 2018, 2019a, b, c, d).

In some practical DMPs, it is difficult for decision-makers (DMs) to give their preference information in quantitative form, but they are easy to describe DMs' opinions with linguistic variables (LVs) (Zadeh 1975; Herrera and Martinez 2000). Then Xu (2004) puts forward linguistic term set (LTS) and continuous linguistic term set (CLTS). Some extended LFSs are widely used in complex DMPs, such as linguistic hesitation fuzzy set (LHFS) (Gou et al. 2018), linguistic neutrosophic set (LNS) (Jin et al. 2019), linguistic intuitionistic fuzzy set (LIFS) (Chen et al. 2015; Arora and Garg 2019; Verma and Sharma 2013; Verma 2014), linguistic Pythagoras fuzzy set (LPFS) (Garg 2018), and so on. Besides, linguistic decisionmaking (Herrera and Herrera-Viedma 2000; Li et al. 2017) and the multi-granularity of binary linguistic (Herrera and Martinez 2000, 2001) are analyzed and studied. In the development of LFSs, Chen et al. (2015) proposed to combine LTS and IFS, and put forward LIFS. He expressed membership degree (MD) and NMD by LTS, and received extensive attention. Correspondingly, a series of AOs dealing with LIFSs are proposed (Garg and Kumar 2018; Liu and Qin 2017; Garg and Kumar 2019; Verma 2016, 2020; Verma and Merigó 2020). Methods of multi-attribute group decision-making in recent 10 years have been summarized and studied (Mohd et al. 2017). However, due to the uncertainty of the DM environment and the limitation of the DM's knowledge, the linguistic information improved by the DM may be uncertain. However, LIFSs only give MD and NMD, and do not give information between them, LIFSs does not explicitly explain uncertainty. In order to solve this problem, Ye (2018) puts forward the theory of linguistic cubic variable (LCV) and its related theories. The theory of LCVs analyzes the satisfied, unsatisfied and uncertain information which can not be explained by the theory of LIFSs. It is a generalization of LFSs or LIFSs. Each element of the LCVs are composed of linguistic MD and linguistic NMD. Linguistic MD is the collection of two terms one is interval-valued fuzzy set while other is fuzzy set. linguistic NMD is also described in the same manner. Therefore, LCVs can show more information in describing practical problems. Cubic fuzzy set (CFS) has more desirable information than FS and IFS (Kaur and Garg 2018a, b, 2019). Some researchers have made many contributions to the research of CFS: Mahmood et al. (2016) extended CFS to the cubic hesitation fuzzy Set, Fahmi et al. (2018a, b) and Lu and Ye (2019) extended CFS to the cubic linguistic hesitation fuzzy set, and so on. Subsequently, some AOs based on CFS are proposed and applied in practice (Fahmi et al. 2017, 2018c, d, 2019; Qiyas and Abdullah 2020).

In the research of AOs, Chen et al. (2015) proposed linguistic intuitionistic fuzzy weighted average (LIFWA) operator, linguistic intuitionistic fuzzy ordered weighted average (LIFOWA) operator and so on. But these AOs have two defects: (1) The operation rules are 
established by a special t-norm (TN) and t-conorm (TC). (2) The aggregation is based on the preference of DM, and the attributes are independent. The related research work is very rich (Meng et al. 2019; Mishra et al. 2019). In addition, copulas and co-copulas are classic examples of TNs and TCs. Copula (Nelsen 1998) reflects the relationship between variables and keeps more original information in the aggregation process. Copula has two characteristics: (1) there are many types of copulas and co-copulas, DMs can choose different types of copula and co-copula according to the actual situation; (2) copula function can reflect the relationship between attributes in DMPs. Although many AOs of LIFSs are proposed to solve DMP (Tao et al. 2018a, b; Chen et al. 2018), they have some limitations. In the theory and application of LICFSs, Muneeza and Abdullah (2020) improved IFSs by using the theory of cubic set, and proposed a series of AOs, but these AOs are based on TN and TC, which have great limitations.

From the above analysis, we can see that LICFSs is a very useful tool in dealing with uncertainty problems. In this paper, the superior characteristics of Copula function are applied to LICFSs and a variety of AOs are constructed. On the one hand, different types of Copula functions are introduced into LICFSs, some theoretical proofs are given, and different AOs are given. On the other hand, parameter analysis is a very important subject, which must select appropriate parameters according to the characteristics of practical problems or functions. Therefore, it is necessary and meaningful to study some problems. For example: What is the expression of LICFSs AO based on Copula function? How the parameters of the AOs are selected? How do parameters affect the sorting results? What are the advantages compared with other AOs?

Therefore, the purpose and motivation of this paper is to combine ECs, ECCs and LICFSs, construct some AOs and study these AOs, and finally solve the selection problem of online education live platform. Based on the above overview and discussion, the main work of this paper is as follows:

(1) to propose the new version of copulas and co-copulas by extending the domain and the range of copulas and co-copulas from $[0,1]$ to $[0, \ell](\ell>0)$;

(2) to define the new operation laws of LICFVs based on ECs and ECCs in order to build the decision-making approaches in the linguistic intuitionistic fuzzy environment;

(3) to propose a family of new LICFCWA AOs and LICFCWG AOs for managing LICFVs by combing proposed new operational rules;

(4) to carry out detailed parameter analysis and comparative experiments.

In order to achieve these, the structure of this paper is as follows: in Sect. 2, some basic definitions of LICFVs are reviewed, and the classic copulas and co-copulas are extended. In Sect. 3, LICFCWA and LICFCWG AOs are proposed. In Sect. 4, a case study is carried out to solve online education live platform selection, and a detailed parameter analysis is carried out. In Sect. 5, in order to illustrate the effectiveness of the methods, a comparative experiment is carried out; the LICFS is reduced to LIFS, and the experimental comparison is made; the LICFS is reduced to ICFS, and the experimental comparison is made, and some advantages of this method are analyzed. Section 6 draws the conclusion of this paper. The graphical abstract of this paper is given (see Fig. 1).

\section{Preliminaries}

In this section, the basic concepts and related properties of the linguistic intuitionistic cubic fuzzy set are given. Copula and co-copula are extended to deal with linguistic information. 


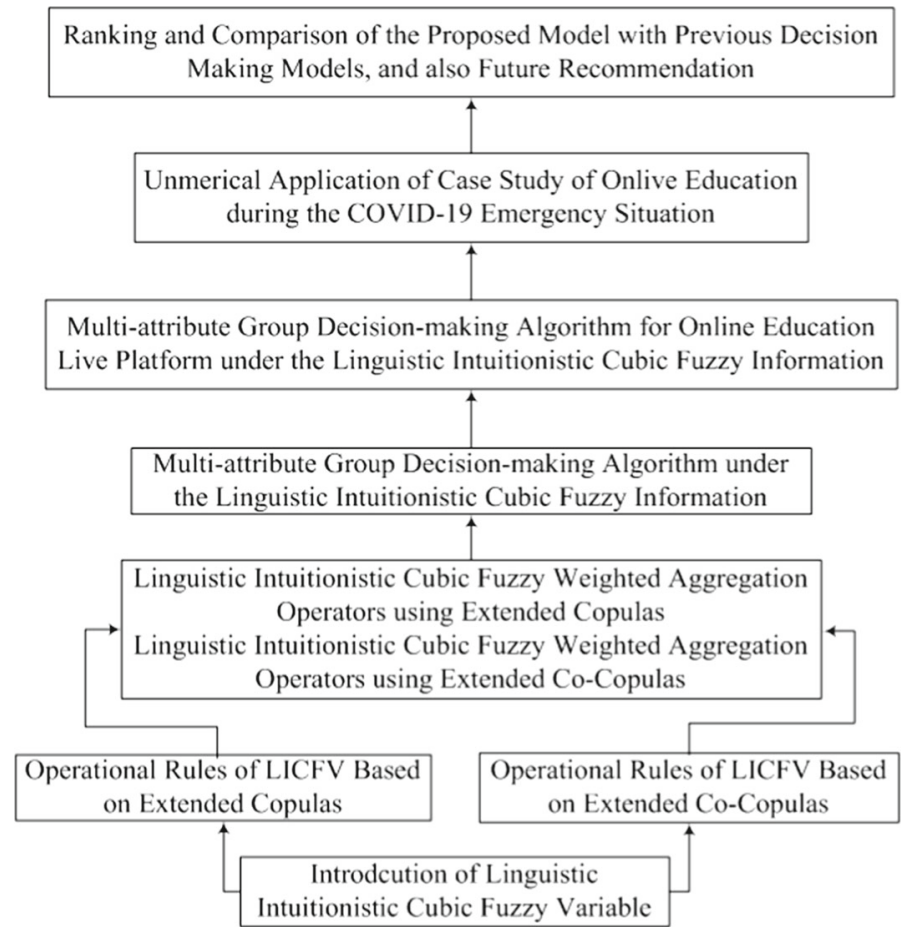

Fig. 1 Graphical abstract

\subsection{Definition of LICFSs}

First of all, we give the basic concepts involved and precious properties of fuzzy set (FS), intuitionistic fuzzy set (IFS), linguistic intuitionistic cubic fuzzy variables (LICFVs), which are the basis of this work.

Definition 1 (Zadeh 1965) Let non-empty set $X$. A FS $F$ in $X$ is defined as follows:

$$
F=\left\{\left(x, \mu_{F}(x)\right) \mid x \in X\right\},
$$

where $\mu_{F(x)}: X \rightarrow[0,1]$ is the membership grade of a FS $F$.

In what follows, $X$ denotes the non-empty set if non-specific.

Definition 2 (Atanassov 1986) An IFS $F$ in $X$ is defined as follows:

$$
F=\left\{\left(x, \mu_{F}(x), v_{F}(x)\right) \mid x \in X\right\},
$$

where $\mu_{F}(x): X \rightarrow[0,1]$ and $v_{F}(x): X \rightarrow[0,1]$, and $0 \leq \mu_{F}(x)+v_{F}(x) \leq 1 . \mu_{F}(x)$ and $v_{F}(x)$ are the MD and NMD of the element $x \in X$, respectively.

In addition, $\pi_{F}(x)=1-\mu_{F}(x)-\nu_{F}(x)$ is called the grade of indeterminacy of $x \in X$ (Atanassov 1986; Szmidt and Kacprzyk 2000).

Definition 3 (Cuong and Phong 2015; Szmidt and Kacprzyk 2000) Let $S=\left(s_{0}, s_{1}, \ldots, s_{\ell}\right)$ be the finite and absolutely order distinct term set. Then $S$ is called the linguistic term set 
(LTS), where $\ell$ is an even number, then $S$ can be written as $S=\left(s_{0}, s_{1}, \ldots, s_{\ell}\right)$. The following characteristics of the $S$ must be satisfied:

(1) Ordered : $s_{i}<s_{j} \Leftrightarrow i<j$;

(2) Negation : neg $\left(s_{i}\right)=s_{\ell-i}$;

(3) Maximum : $s_{i} \geq s_{j} \Leftrightarrow \max \left(s_{i}, s_{j}\right)=s_{i}$;

(4) Minimum : $s_{i} \leq s_{j} \Leftrightarrow \min \left(s_{i}, s_{j}\right)=s_{i}$.

The extended form of the discrete term set $S$ is called a continues linguistic term set (CLTS) and defined as $S^{*}=\left\{s_{e} \mid s_{a} \leq s_{e} \leq s_{b}, e \in[0, \ell]\right\}$, and if $s_{e} \in S^{*}$, then $s_{e}$ is said to be original term, otherwise virtual term.

Definition 4 (Zhang 2014) Let $S^{*}=\left\{s_{e} \mid s_{a} \leq s_{e} \leq s_{b}, e \in[0, \ell]\right\}$ be a CLTS. Then a linguistic intuitionistic fuzzy set (LIFS) is defined as

$$
F=\left\{\left\langle x, s_{\mu}(x), s_{\nu}(x)\right\rangle \mid x \in X\right\},
$$

where $s_{\mu}(x), s_{\nu}(x) \in S^{*}$ stands for the linguistic positive and linguistic negative grades of the element $x \in X$. We shall denote a pair of $\left\langle s_{\mu}(x), s_{\nu}(x)\right\rangle$ as linguistic intuitionistic fuzzy variable.

For any $x \in X$, the condition $\mu+v \leq \ell$ is always satisfied, and $s_{\pi(x)}=s_{\ell-\mu-v}$ is the linguistic refusal grade of $x$ to $X$.

Definition 5 (Muneeza and Abdullah 2020) Let $S^{*}=\left\{s_{e} \mid s_{a} \leq s_{e} \leq s_{b}, e \in[0, \ell]\right\}$ be a CLTS. Then a linguistic intuitionistic cubic fuzzy set (LICFS) $H$ in $X$ is defined as

$$
H=\left\{\left(x,\left\langle\left[s_{\mu^{-}}, s_{\mu^{+}}\right], s_{t}\right\rangle,\left\langle\left[s_{v^{-}}, s_{v^{+}}\right], s_{r}\right\rangle\right) \mid x \in X\right\},
$$

where $\left\langle\left[s_{\mu^{-}}, s_{\mu^{+}}\right], s_{t}\right\rangle,\left\langle\left[s_{v^{-}}, s_{v^{+}}\right], s_{r}\right\rangle$ denote the exact grade of positive and negative membership grade, respectively, which should be satisfied with $\mu^{-} \leq \mu^{+}, v^{-} \leq v^{+}, \mu^{+}+t \leq \ell$, $v^{+}+r \leq \ell$, and $t+r \leq \ell$.

Furthermore, the hesitation margin is defined as

$$
s_{\pi(x)}=\left\{\left\langle\left[s_{\ell-\left(\mu^{+}+v^{+}\right)}, s_{\ell-\left(\mu^{-}+v^{-}\right)}\right], s_{\ell-(t+r)}\right)\right\} .
$$

In Eq. (4), $h_{i}=\left\{\left\langle\left[s_{\mu_{i}^{-}}, s_{\mu_{i}^{+}}\right], s_{t_{i}}\right\rangle,\left\langle\left[s_{v_{i}^{-}}, s_{v_{i}^{+}}\right], s_{r_{i}}\right\rangle\right\}, i=1, \ldots, n$ are called the linguistic intuitionistic cubic fuzzy variables (LICFVs).

Definition 6 Let $h \in H$ be a LICFV. Then score function $\operatorname{Sc}(h)$ is expressed as

$$
\operatorname{Sc}(h)=\frac{\mu^{-}+\mu^{+}+t-v^{-}-v^{+}-r}{6 \ell}, \operatorname{Sc}(h) \in[-1,1] .
$$

Definition 7 Let $h \in H$ be a LICFV. Then accuracy function $\operatorname{Ac}(h)$ is expressed as

$$
\operatorname{Ac}(h)=\frac{\mu^{-}+\mu^{+}+t+v^{-}+v^{+}+r}{6 \ell}, \operatorname{Ac}(h) \in[0,1] .
$$

Definition 8 Let $h_{1}, h_{2} \in H$ be the two LICFVs, their expected values comparison are defined as

(1) $\operatorname{Sc}\left(h_{1}\right)>\operatorname{Sc}\left(h_{2}\right) \Longrightarrow h_{1}>h_{2}$;

(2) $\operatorname{Sc}\left(h_{1}\right)<\operatorname{Sc}\left(h_{2}\right) \Longrightarrow h_{1}<h_{2}$;

(3) $\operatorname{Sc}\left(h_{1}\right)=\operatorname{Sc}\left(h_{2}\right)$ and

(a) $\operatorname{Ac}\left(h_{1}\right)>\operatorname{Ac}\left(h_{2}\right) \Longrightarrow h_{1}>h_{2}$;

(b) $\operatorname{Ac}\left(h_{1}\right)<\operatorname{Ac}\left(h_{2}\right) \Longrightarrow h_{1}<h_{2}$;

(c) $\operatorname{Ac}\left(h_{1}\right)=\operatorname{Ac}\left(h_{2}\right) \Longrightarrow h_{1}=h_{2}$. 


\subsection{Extended copula and extended co-copula}

Copulas and co-copulas are the binary operation on [0, 1]. So they fail to deal with linguistic information. Therefore, it is necessary to extend copula and co-copula to deal with linguistic information.

Definition 9 (Nelsen 1998) A binary function $C:[0, \ell]^{2} \rightarrow[0, \ell]$ is called an extended copulas (ECs) if $C$ fulfills the conditions: for all $x, y, x_{1}, y_{1} \in[0, \ell]$

(1) $C(x, y)+C\left(x_{1}, y_{1}\right) \geq C\left(x, y_{1}\right)+C\left(x_{1}, y\right)$;

(2) $C(x, 0)=C(0, x)=0$;

(3) $C(x, \ell)=C(\ell, x)=x$.

Definition 10 (Nelsen 1998) Let $\sigma:[0, \ell] \rightarrow[0,+\infty)$ and $\psi:[0,+\infty) \rightarrow \sigma:[0, \ell]$. If $\sigma, \psi$ satisfy the following condition, for all $(x, y) \in[0, \ell]^{2}$ :

1. $\sigma$ is continuous;

2. $\sigma$ is strictly decreasing;

3. $\sigma(\ell)=0$;

4. $\psi(x)=\left\{\begin{aligned} \varrho^{-1}(x), & x \in[0, \varrho(0)] \\ 0, & x \in[\varrho(0),+\infty]\end{aligned}\right.$

and

$$
C(x, y)=\psi(\varrho(x)+\varrho(y)) .
$$

The copula $C$ is called extended Archimedean copula (EAC).

The generator $\varrho$ of an EC if a mapping from $[0, \ell]$ to $R^{+}$and $\varrho^{-1}$ is the mapping from $R^{+}$to $[0, \ell]$ with $\varrho(0)=+\infty$ and $\varrho(\ell)=0$. According to Genest and Mackay (1986), the $\mathrm{C}$ can be rewritten as

$$
C(x, y)=\varrho^{-1}(\varrho(x)+\varrho(y)) .
$$

Definition 11 Let $C$ be an EC, for all $(x, y) \in[0, \ell]^{2}$, the new function

$$
C^{*}(x, y)=\ell-C(\ell-x, \ell-y)
$$

is called extended co-copula (ECC).

From the definition of ECC, $C^{*}$ is bounded, But it not an EC. For example, for all $x \in[0, \ell]$, $C^{*}(\ell, x)=\ell-C(\ell-\ell, \ell-x)=\ell$, it follows that $C^{*}$ does not satisfy (Def. 9 (3)).

In what follows, all ECs are all EACs if not specified.

To introduce some new operations for LICFVs based on ECs and ECCs mentioned above, the following conclusion is given first.

Theorem 1 For all $x_{1}, x_{2}, y_{1}, y_{2} \in[0, \ell]$, if $x_{i}+y_{i} \leq \ell(i=1,2)$, then $0 \leq C\left(x_{1}, x_{2}\right)+$ $C^{*}\left(y_{1}, y_{2}\right) \leq \ell$.

The proof of this theorem is the same as the proof process of similar theorem (Tao et al. 2018a), and the proof process is omitted here. Next, we give some special ECs and ECCs depending on the generator.

Case 1. The generator $\varrho(x)=\left(-\ln \left(\frac{x}{\ell}\right)\right)^{\theta}$, where $\varrho^{-1}(x)=\ell e^{-x^{\frac{1}{\theta}}}$ and $\theta \geq 1$. 
It follows from the definition of EC that

$$
\begin{aligned}
C_{G}(x, y) & =\varrho^{-1}(\varrho(x)+\varrho(y)) \\
& =\varrho^{-1}\left(\left(-\ln \left(\frac{x}{\ell}\right)\right)^{\theta}+\left(-\ln \left(\frac{y}{\ell}\right)\right)^{\theta}\right) \\
& =\ell e^{-\left(\left(-\ln \left(\frac{x}{\ell}\right)\right)^{\theta}+\left(-\ln \left(\frac{y}{\ell}\right)\right)^{\theta}\right)^{\frac{1}{\theta}}} .
\end{aligned}
$$

According to the definition of ECC, we have

$$
C_{G}^{*}(x, y)=\ell-\ell e^{-\left(\left(-\ln \left(\frac{\ell-x}{\ell}\right)\right)^{\theta}+\left(-\ln \left(\frac{\ell-y}{\ell}\right)\right)^{\theta}\right)^{\frac{1}{\theta}}} .
$$

When $\theta=1, C_{G}$ and $C_{G}^{*}$ will reduce to extended algebraic $\operatorname{TN} C_{G}(x, y)=\frac{x y}{\ell}$ and extended algebraic $\operatorname{TC} C_{G}^{*}(x, y)=(x+y)-\frac{x y}{\ell}$.

Case 2. The generator $\varrho(x)=\left(\frac{x}{\ell}\right)^{-\theta}-1$, where $\varrho^{-1}(x)=\ell(x+1)^{-\frac{1}{\theta}}, \theta \geq-1$ and $\theta \neq 0$. According to the definition of EC and ECC, they can be as follows:

$$
C_{C}(x, y)=\ell\left(\left(\frac{x}{\ell}\right)^{-\theta}+\left(\frac{y}{\ell}\right)^{-\theta}-1\right)^{-\frac{1}{\theta}}
$$

and

$$
C_{C}^{*}(x, y)=\ell-\ell\left(\left(\frac{\ell-x}{\ell}\right)^{-\theta}+\left(\frac{\ell-y}{\ell}\right)^{-\theta}-1\right)^{-\frac{1}{\theta}}
$$

Case 3. The generator $C_{F}$ be $\varrho(x)=\ln \left(\frac{e^{-\frac{\theta x}{\ell}}-1}{e^{-\theta}-1}\right)$, where $\varrho^{-1}(x)=\left(-\frac{\ell}{\theta}\right) \ln \left(e^{x}\left(e^{-\theta}-1\right)+1\right)$ and $\theta \neq 0$.

According to the definition of EC and ECC, we have

$$
C_{F}(x, y)=\left(-\frac{\ell}{\theta}\right) \ln \left[\frac{\left(e^{-\frac{\theta x}{\ell}}-1\right)\left(e^{-\frac{\theta y}{\ell}}-1\right)}{e^{-\theta}-1}+1\right]
$$

and

$$
C_{F}^{*}(x, y)=\ell+\frac{\ell}{\theta} \ln \left[\frac{\left(e^{-\frac{\theta(\ell-x)}{\ell}}-1\right)\left(e^{-\frac{\theta(\ell-y)}{\ell}}-1\right)}{e^{-\theta}-1}+1\right]
$$

Case 4. The generator $\varrho(x)=\ln \left(\frac{\ell-\theta(\ell-x)}{x}\right)$, where $\varrho^{-1}(x)=\frac{\ell(1-\theta)}{e^{x}-\theta}$, and $\theta \in[-1,1)$.

According to the definition of EC and ECC, they can be as follows:

$$
C_{A}(x, y)=\frac{\ell x y}{\ell^{2}-\theta(\ell-x)(\ell-y)}
$$

and

$$
C_{A}^{*}(x, y)=\ell-\frac{\ell(\ell-x)(\ell-y)}{\ell^{2}-\theta x y} .
$$


Case 5. The generator $\varrho(x)=-\ln \left(1-\left(1-\frac{x}{\ell}\right)^{\theta}\right)$, where $\varrho^{-1}(x)=\ell-\ell\left(1-e^{-x}\right)^{\frac{1}{\theta}}$, and $\theta \geq 1$. According to the definition of EC and ECC, we have

$$
C_{J}(x, y)=\ell-\frac{\left(\ell^{\theta}\left((\ell-x)^{\theta}+(\ell-y)^{\theta}\right)-(\ell-x)^{\theta}(\ell-y)^{\theta}\right)^{\frac{1}{\theta}}}{\ell}
$$

and

$$
C_{J}^{*}(x, y)=\ell\left(x^{\theta}+y^{\theta}-\left(\frac{x y}{\ell}\right)^{\theta}\right)^{\frac{1}{\theta}} .
$$

\section{Aggregation operators on linguistic intuitionistic cubic fuzzy variables}

In this section, the operational laws of LICFVs is given first, then the AOs on LICFVs is given, and the relevant properties are proved. Finally, some different types of AOs are given.

\subsection{Operational laws of linguistic intuitionistic cubic fuzzy variables}

Definition 12 Let $h_{i}=\left\{\left(\left[s_{\mu_{i}^{-}}, s_{\mu_{i}^{+}}\right], s_{t_{i}}\right),\left(\left[s_{v_{i}^{-}}, s_{v_{i}^{+}}\right], s_{r_{i}}\right)\right\},(i=1,2)$ be the two LICFVs. Then the operations of LICFVs based on ECs and ECCs are defined as
(L1) $h_{1} \bigoplus h_{2}=\left\{\left(\left[s_{C^{*}\left(\mu_{1}^{-}, \mu_{2}^{-}\right)}, s_{C^{*}\left(\mu_{1}^{+}, \mu_{2}^{+}\right)}\right], s_{C^{*}\left(t_{1}, t_{2}\right)}\right),\left(\left[s_{C\left(v_{1}^{-}, v_{2}^{-}\right)}, s_{C\left(v_{1}^{+}, v_{2}^{+}\right)}\right]\right.\right.$, $\left.\left.s_{C\left(r_{1}, r_{2}\right)}\right)\right\}$;
(L2) $h_{1} \otimes h_{2}=\left\{\left(\left[s_{C\left(\mu_{1}^{-}, \mu_{2}^{-}\right)}, s_{C\left(\mu_{1}^{+}, \mu_{2}^{+}\right)}\right], s_{C\left(t_{1}, t_{2}\right)}\right),\left(\left[s_{C^{*}\left(v_{1}^{-}, v_{2}^{-}\right)}, s_{C^{*}\left(v_{1}^{+}, v_{2}^{+}\right)}\right]\right.\right.$, $\left.\left.s_{C^{*}\left(r_{1}, r_{2}\right)}\right)\right\}$.

Where $C^{*}(x, y)=\ell-C(\ell-x, \ell-y)$, and $C(x, y)=\varrho^{-1}(\varrho(x)+\varrho(y))$, and $\varrho(x)$ can be any function from Case 1 to Case 5 (See the following for specific analysis).

It is easy to verify that $\bigoplus$ and $\bigotimes$ satisfy associative law, that is, for all three LICFVs $h_{1}, h_{2}, h_{3}$.

(1) $\left(h_{1} \oplus h_{2}\right) \oplus h_{3}=h_{1} \oplus\left(h_{2} \oplus h_{3}\right)$;

(2) $\left(h_{1} \otimes h_{2}\right) \otimes h_{3}=h_{1} \otimes\left(h_{2} \otimes h_{3}\right)$.

For any $\lambda>0$, we can define the following operations:

(L3) $\lambda h=\left\{\left(\left[s_{\ell-\varrho^{-1}\left(\lambda \varrho\left(\ell-\mu^{-}\right)\right)}, s_{\ell-\varrho^{-1}\left(\lambda \varrho\left(\ell-\mu^{+}\right)\right)}\right], s_{\ell-\varrho^{-1}(\lambda \varrho(\ell-t))}\right)\right.$,

$\left.\left(\left[s_{\varrho^{-1}\left(\lambda \varrho\left(v^{-}\right)\right)}, s_{\varrho^{-1}\left(\lambda \varrho\left(v^{+}\right)\right)}\right], s_{\varrho^{-1}(\lambda \varrho(r))}\right)\right\}$

(L4) $h^{\lambda}=\left\{\left(\left[s_{\varrho^{-1}\left(\lambda \varrho\left(v^{-}\right)\right)}, s_{\varrho^{-1}\left(\lambda \varrho\left(v^{+}\right)\right)}\right], s_{\varrho^{-1}(\lambda \varrho(r))}\right)\right.$,

$\left.\left(\left[s_{\left.\ell-\varrho^{-1} \lambda \varrho\left(\ell-\mu^{-}\right)\right)}, s_{\ell-\varrho^{-1}\left(\lambda \varrho\left(\ell-\mu^{+}\right)\right)}\right], s_{\ell-\varrho^{-1}(\lambda \varrho(\ell-t))}\right)\right\}$.

According to the above discussion, for all three LICFVs $h_{1}, h_{2}, h_{3}$, and $p, q>0$, the following laws can be obtained

(3) $p h_{1} \bigoplus q h_{1}=(p+q) h_{1}$;

(4) $h_{1}^{p} \otimes h_{2}^{p}=\left(h_{1} \otimes h_{2}\right)^{p}$;

(5) $h_{1}^{p} \otimes h_{1}^{q}=h_{1}^{p+q}$.

According to the above definitions and theorems, we can easily get the following theorem.

Theorem 2 Let $h_{1}, h_{2}$ be two LICFVs, for $p>0, h_{1} \oplus h_{2}, h_{1} \otimes h_{2}, p h_{1}, h_{1}^{p}$ are all LICFVs. 


\subsection{Aggregation operators on linguistic intuitionistic cubic fuzzy variables}

In this section, it will give a detailed description of LICFVs aggregation operators (linguistic intuitionistic cubic fuzzy extended copula weighted average (LICFCWA) operators and linguistic intuitionistic cubic fuzzy extended copula weighted geometric (LICFCWG) operators) and their properties. Then different forms of ECs are combined, and the concrete operators are given.

Definition 13 Let $h_{i}=\left\{\left(\left[s_{\mu_{i}^{-}}, s_{\mu_{i}^{+}}\right], s_{t_{i}}\right),\left(\left[s_{v_{i}^{-}}, s_{v_{i}^{+}}\right], s_{r_{i}}\right)\right\},(i=1, \ldots, n)$ be the set of LICFVs, and LICFCWA is a mapping LICFCWA: $\Omega^{n} \rightarrow \Omega$,

$$
\operatorname{LICFCWA}\left(h_{1}, \ldots, h_{n}\right)=\bigoplus_{j=1}^{n} w_{i} h_{i},
$$

where $w_{i}$ is the weight of $h_{i}(i=1, \ldots n), 0 \leq w_{i} \leq 1$ and $\sum_{j=1}^{n} w_{i}=1$. Especially, if $w_{i}=\frac{1}{n}$, then the LICFCWA operator becomes an linguistic intuitionistic cubic fuzzy copula averaging operator of dimension $n$. That is to say:

$$
\operatorname{LICFCWA}\left(h_{1}, \ldots, h_{n}\right)=\frac{1}{n}\left(h_{1} \bigoplus \cdots \bigoplus h_{n}\right) .
$$

Theorem 3 Let $h_{i} \in H(i=1, \ldots, n)$ be the set of LICFVs, Then there aggregated value by using the LICFCWA operator is also a LICFVs, and

$$
\begin{aligned}
& \operatorname{LICFCWA}\left(h_{1}, \ldots, h_{n}\right)=\bigoplus_{i=1}^{n} w_{i} h_{i} \\
& =\left\{\left(\left[s_{\ell-\varrho^{-1}\left(\Sigma_{i=1}^{n} w_{i} \varrho\left(\ell-\mu_{i}^{-}\right)\right)}, s_{\ell-\varrho^{-1}\left(\Sigma_{i=1}^{n} w_{i} \varrho\left(\ell-\mu_{i}^{+}\right)\right)}\right], s_{\ell-\varrho^{-1}\left(\Sigma_{i=1}^{n} w_{i} \varrho\left(\ell-t_{i}\right)\right)}\right),\right. \\
& \left.\quad\left(\left[s_{\varrho^{-1}\left(\Sigma_{i=1}^{n} w_{i} \varrho\left(v_{i}^{-}\right)\right)}, s_{\varrho^{-1}\left(\Sigma_{i=1}^{n} w_{i} \varrho\left(v_{i}^{+}\right)\right)}\right], s_{\varrho^{-1}\left(\Sigma_{i=1}^{n} w_{i} \varrho\left(r_{i}\right)\right)}\right)\right\},
\end{aligned}
$$

where $w_{i}$ is the weight of $h_{i}(i=1, \ldots n), 0 \leq w_{i} \leq 1$ and $\sum_{i=1}^{n} w_{i}=1$.

Proof We used the mathematical induction principle to prove this Theorem. (1) If $n=2$, then using the operational laws (L3), we have

$$
\begin{aligned}
w_{1} h_{1}= & \left\{\left(\left[s_{\ell-\varrho^{-1}\left(w_{1} \varrho\left(\ell-\mu_{1}^{-}\right)\right)}, s_{\ell-\varrho^{-1}\left(w_{1} \varrho\left(\ell-\mu_{1}^{+}\right)\right)}\right], s_{\ell-\varrho^{-1}\left(w_{1} \varrho\left(\ell-t_{1}\right)\right)}\right),\right. \\
& \left.\left(\left[s_{\varrho^{-1}\left(w_{1} \varrho\left(v_{1}^{-}\right)\right)}, s_{\varrho^{-1}\left(w_{1} \varrho\left(v_{1}^{+}\right)\right)}\right], s_{\varrho^{-1}\left(w_{1} \varrho\left(r_{1}\right)\right)}\right)\right\} . \\
w_{2} h_{2}=\left\{\left(\left[s_{\ell-\varrho^{-1}\left(w_{2} \varrho\left(\ell-\mu_{2}^{-}\right)\right)}, s_{\ell-\varrho^{-1}\left(w_{2} \varrho\left(\ell-\mu_{2}^{+}\right)\right)}\right], s_{\ell-\varrho^{-1}\left(w_{2} \varrho\left(\ell-t_{2}\right)\right)}\right),\right. & \left.\left(\left[s_{\varrho^{-1}\left(w_{2} \varrho\left(v_{2}^{-}\right)\right)}, s_{\varrho^{-1}\left(w_{2} \varrho\left(v_{2}^{+}\right)\right)}\right], s_{\varrho^{-1}\left(w_{2} \varrho\left(r_{2}\right)\right)}\right)\right\} .
\end{aligned}
$$

Based on the operational law (L1), we have

$$
\begin{aligned}
& \operatorname{LICFCWA}\left(h_{1}, h_{2}\right)=w_{1} h_{1} \bigoplus w_{1} h_{1}
\end{aligned}
$$

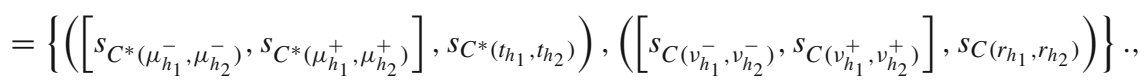

$$
\begin{aligned}
& \text { 基 springer } S \mathrm{D} / \mathrm{MAC}
\end{aligned}
$$


where

$$
\begin{aligned}
& \mu_{h_{i}}^{-}=\ell-\varrho^{-1}\left(w_{i} \varrho\left(\ell-\mu_{i}^{-}\right)\right), \quad \mu_{h_{i}}^{+}=\ell-\varrho^{-1}\left(w_{i} \varrho\left(\ell-\mu_{i}^{+}\right)\right), \\
& t_{h_{i}}=\ell-\varrho^{-1}\left(w_{i} \varrho\left(\ell-t_{i}\right)\right), \quad v_{h_{i}}^{-}=\ell-\varrho^{-1}\left(w_{i} \varrho\left(\ell-v_{i}^{-}\right)\right), \\
& v_{h_{i}}^{+}=\ell-\varrho^{-1}\left(w_{i} \varrho\left(\ell-v_{i}^{+}\right)\right), \quad r_{h_{i}}=\ell-\varrho^{-1}\left(w_{i} \varrho\left(\ell-r_{i}\right)\right) .
\end{aligned}
$$

According to Eqs. (7) and (8), we have

$$
\begin{aligned}
& \varrho\left(\ell-\mu_{h_{i}}^{-}\right)=\varrho\left(\varrho^{-1}\left(w_{i} \varrho\left(\ell-\mu_{i}^{-}\right)\right)\right)=w_{i} \varrho\left(\ell-\mu_{i}^{-}\right), \\
& \varrho\left(v_{h_{i}}^{-}\right)=\varrho\left(\varrho^{-1}\left(w_{i} \varrho\left(v_{i}^{-}\right)\right)\right)=w_{i} \varrho\left(v_{i}^{-}\right)
\end{aligned}
$$

and

$$
\begin{aligned}
C^{*}\left(\mu_{h_{1}}^{-}, \mu_{h_{2}}^{-}\right) & =\ell-C\left(\ell-\mu_{h_{1}}^{-}, \ell-\mu_{h_{2}}^{1}\right) \\
& =\ell-\varrho^{-1}\left[\varrho\left(\ell-\mu_{h_{1}}^{-}\right)+\varrho\left(\ell-\mu_{h_{2}}^{-}\right)\right] \\
& =\ell-\varrho^{-1}\left[w_{1} \varrho\left(\ell-\mu_{1}^{-}\right)+w_{2} \varrho\left(\ell-\mu_{2}^{-}\right)\right] \\
& =\ell-\varrho^{-1}\left[\Sigma_{i=1}^{2} w_{i} \varrho\left(\ell-\mu_{i}^{-}\right)\right] .
\end{aligned}
$$

Based on a similar calculation, the following can be drawn:

$$
\begin{aligned}
& C^{*}\left(\mu_{h_{1}}^{+}, \mu_{h_{2}}^{+}\right)=\ell-\varrho^{-1}\left[\Sigma_{i=1}^{2} w_{i} \varrho\left(\ell-\mu_{i}^{+}\right)\right], \quad C^{*}\left(t_{h_{1}}, t_{h_{2}}\right)=\ell-\varrho^{-1}\left[\Sigma_{i=1}^{2} w_{i} \varrho\left(\ell-t_{i}\right)\right], \\
& C\left(v_{h_{1}}^{-}, v_{h_{2}}^{-}\right)=\varrho^{-1}\left[\Sigma_{i=1}^{2} w_{i} \varrho\left(v_{i}^{-}\right)\right], \quad C\left(v_{h_{1}}^{+}, v_{h_{2}}^{+}\right)=\varrho^{-1}\left[\Sigma_{i=1}^{2} w_{i} \varrho\left(v_{i}^{+}\right)\right], \\
& C\left(r_{h_{1}}, r_{h_{2}}\right)=\ell-\varrho^{-1}\left[\Sigma_{i=1}^{2} w_{i} \varrho\left(r_{i}\right)\right] .
\end{aligned}
$$

For the convenience of writing, we denote

$$
g(a, b, k)=\ell-\varrho^{-1}\left[\Sigma_{i=1}^{k} a_{i} \varrho(\ell-b)\right], \quad f(a, b, k)=\varrho^{-1}\left[\Sigma_{i=1}^{k} a_{i} \varrho(b)\right]
$$

where $a$ is the sign of the weight variable, $b$ is the subscript of the linguistic variable, and $k$ is the number of LICVs participating in the aggregation. Therefore,

$$
\begin{aligned}
\operatorname{LICFCWA}\left(h_{1}, h_{2}\right)= & w_{1} h_{1} \bigoplus w_{2} h_{2} \\
= & \left\{\left(\left[s_{g\left(w, \mu_{i}^{-}, 2\right)}, s_{g\left(w, \mu_{i}^{+}, 2\right)}\right], s_{g\left(w, t_{i}, 2\right)}\right),\right. \\
& \left.\left(\left[s_{f\left(w, v_{i}^{-}, 2\right)}, s_{f\left(w, v_{i}^{-}, 2\right)}\right], s_{f\left(w, r_{i}, 2\right)}\right)\right\} .
\end{aligned}
$$

(2) If $n=k$, it can be drawn

$$
\begin{aligned}
& \operatorname{LICFCWA}\left(h_{1}, \ldots, h_{k}\right)=w_{1} h_{1} \bigoplus \cdots \bigoplus w_{k} h_{k} \\
& \quad=\left\{\left(\left[s_{g\left(w, \mu_{i}^{-}, k\right)}, s_{g\left(w, \mu_{i}^{+}, k\right)}\right], s_{g\left(w, t_{i}, k\right)}\right),\left(\left[s_{f\left(w, v_{i}^{-}, k\right)}, s_{f\left(w, v_{i}^{-}, k\right)}\right], s_{f\left(w, r_{i}, k\right)}\right)\right\} .
\end{aligned}
$$


(3) If $n=k+1$, we have

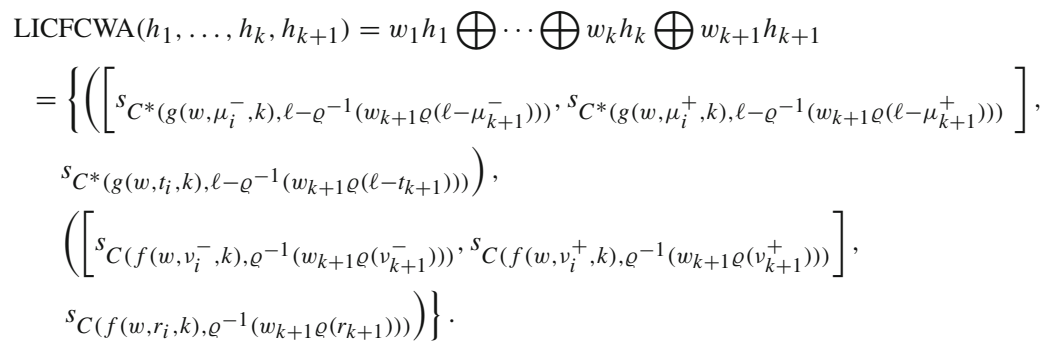

Then according to Eqs. (7) and (8), they are obtained as follows:

$$
\begin{aligned}
& C^{*}\left(g\left(w, \mu_{i}^{-}, k\right), \ell-\varrho^{-1}\left(w_{k+1} \varrho\left(\ell-\mu_{k+1}^{-}\right)\right)\right) \\
& =C^{*}\left(\ell-\varrho^{-1}\left[\Sigma_{i=1}^{k} w_{i} \varrho\left(\ell-\mu_{i}^{-}\right)\right], \ell-\varrho^{-1}\left(w_{k+1} \varrho\left(\ell-\mu_{k+1}^{-}\right)\right)\right) \\
& =\ell-\varrho^{-1}\left[\varrho\left(\varrho^{-1}\left(\Sigma_{i=1}^{k} w_{i} \varrho\left(\ell-\mu_{i}^{-}\right)\right)\right)+\varrho\left(\varrho^{-1}\left(w_{k+1} \varrho\left(\ell-\mu_{k+1}^{-}\right)\right)\right)\right] \\
& \left.=\ell-\varrho^{-1}\left[\Sigma_{i=1}^{k} w_{i} \varrho\left(\ell-\mu_{i}^{-}\right)\right)+w_{k+1} \varrho\left(\ell-\mu_{k+1}^{-}\right)\right] \\
& \left.=\ell-\varrho^{-1}\left[\Sigma_{i=1}^{k+1} w_{i} \varrho\left(\ell-\mu_{i}^{-}\right)\right)\right] .
\end{aligned}
$$

According to similar calculation, we can get

$$
\begin{aligned}
& \left.C^{*}\left(g\left(w, \mu_{i}^{+}, k\right), \ell-\varrho^{-1}\left(w_{k+1} \varrho\left(\ell-\mu_{k+1}^{+}\right)\right)\right)=\ell-\varrho^{-1}\left[\Sigma_{i=1}^{k+1} w_{i} \varrho\left(\ell-\mu_{i}^{+}\right)\right)\right], \\
& \left.C^{*}\left(g\left(w, t_{i}, k\right), \ell-\varrho^{-1}\left(w_{k+1} \varrho\left(\ell-t_{k+1}\right)\right)\right)=\ell-\varrho^{-1}\left[\Sigma_{i=1}^{k+1} w_{i} \varrho\left(\ell-t_{i}\right)\right)\right], \\
& \left.C\left(f\left(w, v_{i}^{-}, k\right), \varrho^{-1}\left(w_{k+1} \varrho\left(v_{k+1}^{-}\right)\right)\right)=\varrho^{-1}\left[\Sigma_{i=1}^{k+1} w_{i} \varrho\left(v_{i}^{-}\right)\right)\right], \\
& \left.C\left(f\left(w, v_{i}^{+}, k\right), \varrho^{-1}\left(w_{k+1} \varrho\left(v_{k+1}^{+}\right)\right)\right)=\varrho^{-1}\left[\Sigma_{i=1}^{k+1} w_{i} \varrho\left(v_{i}^{+}\right)\right)\right], \\
& \left.C\left(f\left(w, r_{i}, k\right), \varrho^{-1}\left(w_{k+1} \varrho\left(r_{k+1}\right)\right)\right)=\varrho^{-1}\left[\Sigma_{i=1}^{k+1} w_{i} \varrho\left(r_{i}\right)\right)\right] .
\end{aligned}
$$

From the above mentioned, we have

$$
\begin{aligned}
& \operatorname{LICFCWA}\left(h_{1}, \ldots, h_{n}\right)=w_{1} h_{1} \bigoplus \cdots \bigoplus w_{n} h_{n} \\
& \quad=\left\{\left(\left[s_{g\left(w, \mu_{i}^{-}, n\right)}, s_{\left(g\left(w, \mu_{i}^{+}, n\right)\right.}\right], s_{g\left(w, t_{i}, n\right)}\right),\left(\left[s_{f\left(w, v_{i}^{-}, n\right)}, s_{f\left(w, v_{i}^{+}, n\right)}\right], s_{f\left(w, r_{i}, n\right)}\right)\right\},
\end{aligned}
$$

where

$$
\begin{aligned}
& \left.g\left(w, \mu_{i}^{-}, n\right)=\ell-\varrho^{-1}\left[\Sigma_{i=1}^{k+1} w_{i} \varrho\left(\ell-\mu_{i}^{-}\right)\right)\right], \\
& \left.f\left(w, v_{i}^{-}, n\right)=\varrho^{-1}\left[\Sigma_{i=1}^{k+1} w_{i} \varrho\left(v_{i}^{-}\right)\right)\right], \\
& \left.g\left(w, \mu_{i}^{+}, n\right)=\ell-\varrho^{-1}\left[\Sigma_{i=1}^{k+1} w_{i} \varrho\left(\ell-\mu_{i}^{+}\right)\right)\right], \\
& \left.f\left(w, v_{i}^{+}, n\right)=\varrho^{-1}\left[\Sigma_{i=1}^{k+1} w_{i} \varrho\left(v_{i}^{+}\right)\right)\right], \\
& \left.g\left(w, t_{i}, n\right)=\ell-\varrho^{-1}\left[\Sigma_{i=1}^{k+1} w_{i} \varrho\left(\ell-t_{i}\right)\right)\right], \\
& \left.f\left(w, r_{i}, n\right)=\varrho^{-1}\left[\Sigma_{i=1}^{k+1} w_{i} \varrho\left(r_{i}\right)\right)\right] .
\end{aligned}
$$


Through the above proof, it can known that Eq. (21) is valid for any $n$. Next, we will discuss some related properties of the LICFCWA. We can get the following inference.

Theorem 4 (Idempotency) Let $h, h_{i} \in H,(i=1, \ldots, n)$ be the set of LICFVs, and all $h_{i}$ are equal to $h$, then

$$
\operatorname{LICFCWA}\left(h_{1}, \ldots, h_{n}\right)=h .
$$

Proof According to all $h_{i}$ are equal, it can be seen that all $\mu_{i}^{-}$are equal. For any $i \in n$, we have

$$
\begin{aligned}
g\left(w, \mu_{i}^{-}, n\right) & =\ell-\varrho^{-1}\left[\Sigma_{i=1}^{n} w_{i} \varrho\left(\ell-\mu_{i}^{-}\right)\right] \\
& =\ell-\varrho^{-1}\left[w_{1} \varrho\left(\ell-\mu_{i}^{-}\right)+w_{2} \varrho\left(\ell-\mu_{i}^{-}\right)+\cdots+w_{3} \varrho\left(\ell-\mu_{i}^{-}\right)\right] \\
& =\ell-\varrho^{-1}\left[\left(\Sigma_{i=1}^{n} w_{i}\right) \varrho\left(\ell-\mu_{i}^{-}\right)\right] \\
& =\ell-\varrho^{-1}\left[\varrho\left(\ell-\mu_{i}^{-}\right)\right] \\
& =\mu_{i}^{-} .
\end{aligned}
$$

According to similar calculation, for any $i \in n$, we can get

$$
\begin{aligned}
& g\left(w, \mu_{i}^{+}, n\right)=\mu_{i}^{+}, g\left(w, t_{i}, n\right)=t_{i}, \\
& f\left(w, v_{i}^{-}, n\right)=v_{i}^{-}, f\left(w, v_{i}^{+}, n\right)=v_{i}^{+}, f\left(w, r_{i}, n\right)=r_{i} .
\end{aligned}
$$

So, we have

$$
\begin{aligned}
& \operatorname{LICFCWA}\left(h_{1}, \ldots, h_{n}\right) \\
& \quad=\left\{\left(\left[s_{g\left(w, \mu_{i}^{-}, n\right)}, s_{\left(g\left(w, \mu_{i}^{+}, n\right)\right.}\right], s_{g\left(w, t_{i}, n\right)}\right),\left(\left[s_{f\left(w, v_{i}^{-}, n\right)}, s_{f\left(w, v_{i}^{+}, n\right)}\right], s_{f\left(w, r_{i}, n\right)}\right)\right\} \\
& \left.=\left\{\left(\left[s_{\mu_{i}^{-}}, s_{\mu_{i}^{+}}\right], s_{t_{i}}\right),\left(\left[s_{v_{i}^{-}}, s_{v_{i}^{+}}\right)\right], s_{r_{i}}\right)\right\} .
\end{aligned}
$$

So,

$$
\operatorname{LICFCWA}\left(h_{1}, \ldots, h_{n}\right)=h .
$$

Theorem 5 (Boundary) Let

$$
h^{-}=\left\{\left(\left[\min \left(s_{\mu_{i}^{-}}\right), \min \left(s_{\mu_{i}^{+}}\right)\right], \min \left(s_{t_{i}}\right)\right),\left(\left[\max \left(s_{\mu_{i}^{-}}\right), \max \left(s_{\mu_{i}^{+}}\right)\right], \max \left(s_{t_{i}}\right)\right)\right\}
$$

and

$$
h^{+}=\left\{\left(\left[\max \left(s_{v_{i}^{-}}\right), \max \left(s_{v_{i}^{+}}\right)\right], \max \left(s_{r_{i}}\right)\right),\left(\left[\min \left(s_{v_{i}^{-}}\right), \min \left(s_{v_{i}^{+}}\right)\right], \min \left(s_{r_{i}}\right)\right)\right\}
$$

be the set of LICFVs for every $i \in n$. Then

$$
h^{-} \leq \operatorname{LICFCWA}\left(h_{1}, \ldots, h_{n}\right) \leq h^{+} .
$$

Proof According to the conditions, the minimum of LICFVs are $h^{-}$and the maximum are $h^{+}$.

So $h^{-} \leq h_{i} \leq h^{+},(i=1, \ldots, n)$, and $\sum_{i=1}^{n} w_{i} h^{-} \leq \sum_{i=1}^{n} w_{i} h_{i} \leq \sum_{i=1}^{n} w_{i} h^{+}$. According to the idempotency, there is $h^{-} \leq \sum_{i=1}^{n} w_{i} h_{i} \leq h^{+}$. So, $h^{-} \leq \operatorname{LICFCWA}\left(h_{1}, \ldots, h_{n}\right) \leq$ $h^{+}$. 
Theorem 6 (Monotonicity) Let $h_{i}^{*}=\left\{\left(\left[s_{\mu_{i}^{-}}^{*}, s_{\mu_{i}^{+}}^{*}\right], s_{t_{i}}^{*}\right),\left(\left[s_{v_{i}^{-}}^{*}, s_{v_{i}^{+}}^{*}\right], s_{r_{i}}^{*}\right)\right\},(i=1, \ldots, n)$ be the set of LICFVs.

Let $\left[s_{\mu_{i}^{-}}, s_{\mu_{i}^{+}}\right] \leq\left[s_{\mu_{i}^{-}}^{*}, s_{\mu_{i}^{+}}^{*}\right], s_{t_{i}} \leq s_{t_{i}}^{*},\left[s_{v_{i}^{-}}^{*}, s_{v_{i}^{+}}^{*}\right] \leq\left[s_{v_{i}^{-}}, s_{v_{i}^{+}}\right], s_{r_{i}}^{*} \leq s_{r_{i}}, i \in n$. Then

$$
\operatorname{LICFCWA}\left(h_{1}, \ldots, h_{n}\right) \leq \operatorname{LICFCWA}\left(h_{1}^{*}, \ldots, h_{n}^{*}\right) .
$$

Proof According to the conditions, for any $i \in n$, we have $h \leq h^{*}$, so, $\sum_{i=1}^{n} w_{i} h_{i} \leq$ $\sum_{i=1}^{n} w_{i} h_{i}^{*}$. So $\operatorname{LICFCWA}\left(h_{1}, \ldots, h_{n}\right) \leq \operatorname{LICFCWA}\left(h_{1}^{*}, \ldots, h_{n}^{*}\right)$.

Definition 14 Let $h_{i} \in H,(i=1, \ldots, n)$ be the set of LICFVs, and LICFCWG is a mapping LICFCWG : $\Omega^{n} \rightarrow \Omega$,

$$
\operatorname{LICFCWG}\left(h_{1}, \ldots, h_{n}\right)=\bigotimes_{j=1}^{n} h_{i}^{w_{i}}
$$

where $w_{i}$ is the weight of $h_{i}(i=1, \ldots n), 0 \leq w_{i} \leq 1$ and $\sum_{j=1}^{n} w_{i}=1$. Especially, if $w_{i}=\frac{1}{n}$, then the LICFCWG operator is become an linguistic intuitionistic cubic fuzzy copula geometric operator of dimension $n$. That is to say:

$$
\operatorname{LICFCG}\left(h_{1}, \ldots, h_{n}\right)=\left(h_{1} \bigotimes \cdots \bigotimes h_{n}\right)^{\frac{1}{n}}
$$

Theorem 7 Let $h_{i}(i=1, \ldots, n)$ be the set of LICFVs. Then there aggregated value by using the LICFCWG operator is also a LICFV, and

$$
\begin{aligned}
\operatorname{LICFCWG}\left(h_{1}, \ldots, h_{n}\right)=\bigotimes_{j=1}^{n} h_{i}^{w_{i}} \\
=\left\{\left(\left[s_{\varrho^{-1}\left(\Sigma_{i=1}^{n} w_{i} \varrho\left(\mu_{i}^{-}\right)\right)}, s_{\varrho^{-1}\left(\Sigma_{i=1}^{n} w_{i} \varrho\left(\mu_{i}^{+}\right)\right)}\right], s_{\varrho^{-1}\left(\Sigma_{i=1}^{n} w_{i} \varrho\left(t_{i}\right)\right)}\right),\right. \\
\left.\quad\left(\left[s_{\ell-\varrho^{-1}\left(\Sigma_{i=1}^{n} w_{i} \varrho\left(\ell-v_{i}^{-}\right)\right)}, s_{\ell-\varrho^{-1}\left(\Sigma_{i=1}^{n} w_{i} \varrho\left(\ell-v_{i}^{+}\right)\right)}\right], s_{\ell-\varrho^{-1}\left(\Sigma_{i=1}^{n} w_{i} \varrho\left(\ell-r_{i}\right)\right)}\right)\right\},
\end{aligned}
$$

where $w_{i}$ is the weight of $h_{i}(i=1, \ldots n), 0 \leq w_{i} \leq 1$ and $\sum_{i=1}^{n} w_{i}=1$.

The proof of this theorem is same as Theorem 5. Similar to the proofs of the properties of LICFCWA, it is easy to obtain the properties of the LICFCWG.

Theorem 8 (Idempotency) Let $h, h_{i} \in H(i=1, \ldots, n)$ be the set of LICFVs, and all $h_{i}$ are equal to $h$, then

$$
\operatorname{LICFCWG}\left(h_{1}, \ldots, h_{n}\right)=h .
$$

Theorem 9 (Boundary) Let

$$
h^{-}=\left\{\left(\left[\min \left(s_{\mu_{i}^{-}}\right), \min \left(s_{\mu_{i}^{+}}\right)\right], \min \left(s_{t_{i}}\right)\right),\left(\left[\max \left(s_{\mu_{i}^{-}}\right), \max \left(s_{\mu_{i}^{+}}\right)\right], \max \left(s_{t_{i}}\right)\right)\right\}
$$

and

$$
h^{+}=\left\{\left(\left[\max \left(s_{v_{i}^{-}}\right), \max \left(s_{v_{i}^{+}}\right)\right], \max \left(s_{r_{i}}\right)\right),\left(\left[\min \left(s_{v_{i}^{-}}\right), \min \left(s_{v_{i}^{+}}\right)\right], \min \left(s_{r_{i}}\right)\right)\right\}
$$

be the set of LICFVs for every $i \in n$. Then

$$
h^{-} \leq \operatorname{LICFCWG}\left(h_{1}, \ldots, h_{n}\right) \leq h^{+}
$$


Theorem 10 (Montotonicity) Let $h_{i}^{*}=\left\{\left(\left[s_{\mu_{i}^{-}}^{*}, s_{\mu_{i}^{+}}^{*}\right], s_{t_{i}}^{*}\right),\left(\left[s_{v_{i}^{-}}^{*}, s_{v_{i}^{+}}^{*}\right], s_{r_{i}}^{*}\right)\right\}, \quad(i=$ $1, \ldots, n)$ be the set of LICFVs.

Let $\left[s_{\mu_{i}^{-}}, s_{\mu_{i}^{+}}\right] \leq\left[s_{\mu_{i}^{-}}^{*}, s_{\mu_{i}^{+}}^{*}\right], s_{t_{i}} \leq s_{t_{i}}^{*},\left[s_{v_{i}^{-}}^{*}, s_{v_{i}^{+}}^{*}\right] \leq\left[s_{v_{i}^{-}}, s_{v_{i}^{+}}\right], s_{r_{i}}^{*} \leq s_{r_{i}}, i \in n$. Then

$$
\operatorname{LICFCWG}\left(h_{1}, \ldots, h_{n}\right) \leq \operatorname{LICFCWG}\left(h_{1}^{*}, \ldots, h_{n}^{*}\right)
$$

\subsection{Some different types of aggregation operators}

In this subsection, we will discuss some special cases of LICFVs AOs.

Case 1. The generator $\varrho(x)=\left(-\ln \left(\frac{x}{\ell}\right)\right)^{\theta}$, where $\varrho^{-1}(x)=\ell e^{-x^{\frac{1}{\theta}}}$ and $\theta \geq 1$.

$$
\operatorname{LICFC}_{\mathrm{G}} \mathrm{WA}\left(h_{1}, \ldots, h_{n}\right)=\bigoplus_{i=1}^{n} w_{i} h_{i}=\left\{\left(\left[s_{u^{-}}, s_{u^{+}}\right], s_{t}\right),\left(\left[s_{v^{-}}, s_{v^{+}}\right], s_{r}\right)\right\},
$$

where

$$
\begin{aligned}
& u^{-}=\ell-\ell e^{-\left(\sum_{i=1}^{n} w_{i}\left(-\ln \left(\frac{\ell-\mu_{i}^{-}}{\ell}\right)\right)^{\theta}\right)^{\frac{1}{\theta}}}, u^{+}=\ell-\ell e^{-\left(\sum_{i=1}^{n} w_{i}\left(-\ln \left(\frac{\ell-\mu_{i}^{+}}{\ell}\right)\right)^{\theta}\right)^{\frac{1}{\theta}}} \\
& v^{-}=\ell e^{-\left(\sum_{i=1}^{n} w_{i}\left(-\ln \left(\frac{v_{i}^{-}}{\ell}\right)\right)^{\theta}\right)^{\frac{1}{\theta}}}, \quad v^{+}=\ell e^{-\left(\sum_{i=1}^{n} w_{i}\left(-\ln \left(\frac{v_{i}^{+}}{\ell}\right)\right)^{\theta}\right)^{\frac{1}{\theta}}} \\
& t=\ell-\ell e^{-\left(\sum_{i=1}^{n} w_{i}\left(-\ln \left(\frac{\ell-t}{\ell}\right)\right)^{\theta}\right)^{\frac{1}{\theta}}}, \quad r=\ell e^{-\left(\sum_{i=1}^{n} w_{i}\left(-\ln \left(\frac{r}{\ell}\right)\right)^{\theta}\right)^{\frac{1}{\theta}}} \cdot \\
& \operatorname{LICFC}_{\mathrm{G}} \mathrm{WG}\left(h_{1}, \ldots, h_{n}\right)=\bigotimes_{i=1}^{n} w_{i} h_{i}=\left\{\left(\left[s_{u^{-}}, s_{u^{+}}\right], s_{t}\right),\left(\left[s_{v^{-}}, s_{v^{+}}\right], s_{r}\right)\right\},
\end{aligned}
$$

where

$$
\begin{aligned}
& u^{-}=\ell e^{-\left(\sum_{i=1}^{n} w_{i}\left(-\ln \left(\frac{\mu_{i}^{-}}{\ell}\right)\right)^{\theta}\right)^{\frac{1}{\theta}},} u^{+}=\ell e^{-\left(\sum_{i=1}^{n} w_{i}\left(-\ln \left(\frac{\mu_{i}^{+}}{\ell}\right)\right)^{\theta}\right)^{\frac{1}{\theta}}} \\
& v^{-}=\ell-\ell e^{-\left(\sum_{i=1}^{n} w_{i}\left(-\ln \left(\frac{\ell-v_{i}^{-}}{\ell}\right)\right)^{\theta}\right)^{\frac{1}{\theta}}}, v^{+}=\ell-\ell e^{-\left(\sum_{i=1}^{n} w_{i}\left(-\ln \left(\frac{\ell-v_{i}^{+}}{\ell}\right)\right)^{\theta}\right)^{\frac{1}{\theta}}} \\
& t=\ell e^{-\left(\sum_{i=1}^{n} w_{i}\left(-\ln \left(\frac{t}{\ell}\right)\right)^{\theta}\right)^{\frac{1}{\theta}}}, \quad r=\ell-\ell e^{-\left(\sum_{i=1}^{n} w_{i}\left(-\ln \left(\frac{\ell-r}{\ell}\right)\right)^{\theta}\right)^{\frac{1}{\theta}}} .
\end{aligned}
$$

Case 2. The generator $\varrho(x)=\left(\frac{x}{\ell}\right)^{-\theta}-1$, where $\varrho^{-1}(x)=\ell(x+1)^{-\frac{1}{\theta}}, \theta \geq-1$ and $\theta \neq 0$.

$$
\operatorname{LICFC}_{C} \operatorname{WA}\left(h_{1}, \ldots, h_{n}\right)=\bigoplus_{i=1}^{n} w_{i} h_{i}=\left\{\left(\left[s_{u^{-}}, s_{u^{+}}\right], s_{t}\right),\left(\left[s_{v^{-}}, s_{v^{+}}\right], s_{r}\right)\right\},
$$


where

$$
\begin{aligned}
& u^{-}=\ell-\ell\left(\sum_{i=1}^{n} w_{i}\left(\left(\frac{\ell-\mu_{i}^{-}}{\ell}\right)^{-\theta}-1\right)+1\right)^{-\frac{1}{\theta}}, v^{-}=\ell\left(\sum_{i=1}^{n} w_{i}\left(\left(\frac{v_{i}^{-}}{\ell}\right)^{-\theta}-1\right)+1\right)^{-\frac{1}{\theta}} \\
& u^{+}=\ell-\ell\left(\sum_{i=1}^{n} w_{i}\left(\left(\frac{\ell-\mu_{i}^{+}}{\ell}\right)^{-\theta}-1\right)+1\right)^{-\frac{1}{\theta}}, v^{+}=\ell\left(\sum_{i=1}^{n} w_{i}\left(\left(\frac{v_{i}^{+}}{\ell}\right)^{-\theta}-1\right)+1\right)^{-\frac{1}{\theta}} \\
& t=\ell-\ell\left(\sum_{i=1}^{n} w_{i}\left(\left(\frac{\ell-t}{\ell}\right)^{-\theta}-1\right)+1\right)^{-\frac{1}{\theta}}, r=\ell\left(\sum_{i=1}^{n} w_{i}\left(\left(\frac{r}{\ell}\right)^{-\theta}-1\right)+1\right)^{-\frac{1}{\theta}} . \\
& \operatorname{LICFC}_{C} \operatorname{WG}\left(h_{1}, \ldots, h_{n}\right)=\bigotimes_{i=1}^{n} w_{i} h_{i}=\left\{\left(\left[s_{u^{-}}, s_{u^{+}}\right], s_{t}\right),\left(\left[s_{v^{-}}, s_{v^{+}}\right], s_{r}\right)\right\}
\end{aligned}
$$

where

$$
\begin{aligned}
& u^{-}=\ell\left(\sum_{i=1}^{n} w_{i}\left(\left(\frac{\mu_{i}^{-}}{\ell}\right)^{-\theta}-1\right)+1\right)^{-\frac{1}{\theta}}, v^{-}=\ell-\ell\left(\sum_{i=1}^{n} w_{i}\left(\left(\frac{\ell-v_{i}^{-}}{\ell}\right)^{-\theta}-1\right)+1\right)^{-\frac{1}{\theta}} \\
& u^{+}=\ell\left(\sum_{i=1}^{n} w_{i}\left(\left(\frac{\mu_{i}^{+}}{\ell}\right)^{-\theta}-1\right)+1\right)^{-\frac{1}{\theta}}, v^{+}=\ell-\ell\left(\sum_{i=1}^{n} w_{i}\left(\left(\frac{\ell-v_{i}^{+}}{\ell}\right)^{-\theta}-1\right)+1\right)^{-\frac{1}{\theta}} \\
& t=\ell\left(\sum_{i=1}^{n} w_{i}\left(\left(\frac{t}{\ell}\right)^{-\theta}-1\right)+1\right)^{-\frac{1}{\theta}}, r=\ell-\ell\left(\sum_{i=1}^{n} w_{i}\left(\left(\frac{\ell-r}{\ell}\right)^{-\theta}-1\right)+1\right)^{-\frac{1}{\theta}} .
\end{aligned}
$$

According to the same method, we can give the concrete AOs of Case 3 ( IICFC $_{F} W A$, LICFC $_{F} W G$ ), Case 4 (LICFC $\left._{\mathrm{A}} \mathrm{WA}, \mathrm{LICFC}_{\mathrm{A}} \mathrm{WG}\right)$ and Case 5 (LICFC WA, LICFC $\mathrm{J}$ WG). It will not be detailed here.

\section{Approach for MAGDM problem with linguistic intuitionistic cubic fuzzy information}

In this section, the general form of MAGDM problem would be shown, and then the algorithm of MAGDM problem with LICFI are designed based on the proposed AOs.

\subsection{General form of MAGDM problem}

In general, a MAGDM problem consists of four parts: the set of decision-makers (DMs): $D=\left\{D^{1}, \ldots, D^{n}\right\}$, weight vector of DMs $\lambda=\left(\lambda_{1}, \ldots, \lambda_{t}\right)$, where $\sum_{t=1}^{n} \lambda_{t}=1$, and $\lambda_{t} \in[0,1]$. Alternatives set $B=$ $\left\{B_{1}, \ldots, B_{k}\right\}$, criteria set $Y=\left\{Y_{1}, \ldots, Y_{m}\right\}$, and weight vector of criteria $w=\left(w_{1}, \ldots, w_{m}\right)$, where $\sum_{i=1}^{m} w_{i}=1$, and $w_{i} \in[0,1]$. Thus, a MAGDM problem can be concisely expressed in LICFVs decision matrix $D^{t},(t=1, \ldots, n)$.

$$
D^{t}=\left(h_{i j}^{t}\right)_{m \times k}=\left\{\left(\left[s_{\mu_{i j}^{-}}, s_{\mu_{i j}^{+}}\right], s_{t_{i j}}\right),\left(\left[s_{v_{i j}^{-}}, s_{v_{i j}^{+}}\right], s_{r_{i j}}\right)\right\}_{m \times k}
$$

Where, $\left(\left[s_{\mu_{i j}^{-}}, s_{\mu_{i j}^{+}}\right], s_{t_{i j}}\right)$ is the exact grade of the positive membership degree of alternative $B_{j}$ satisfying criterion $Y_{i} \cdot\left(\left[s_{v_{i j}^{-}}, s_{v_{i j}^{+}}\right], s_{r_{i j}}\right)$ is the exact grade of the negative membership degree of alternative $B_{j}$ satisfying criterion $Y_{i}$. In addition, the following conditions are met: $\left[\mu_{i j}^{-}, \mu_{i j}^{+}\right],\left[v_{i j}^{-}, v_{i j}^{+}\right] \subset[0, \ell]$, and $s_{t_{i j}}, s_{r_{i j}} \in S^{*}$. 
Fig. 2 Flowchart of MAGDM

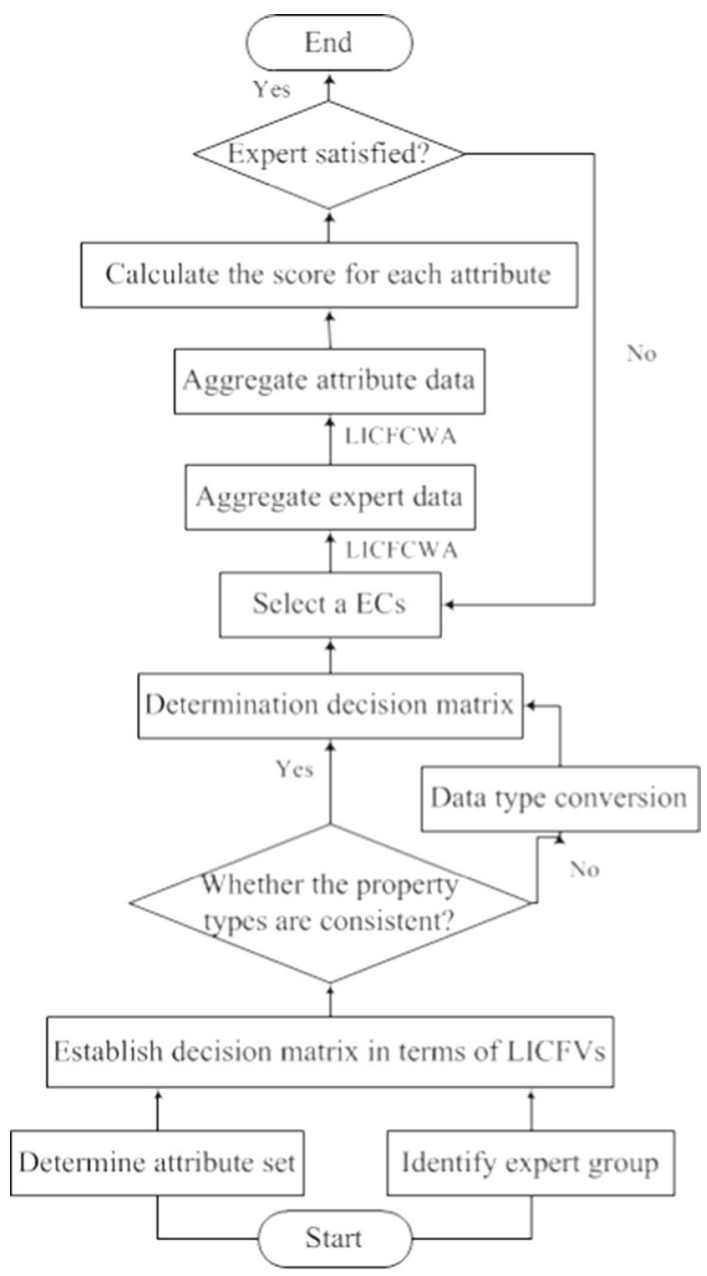

\subsection{Algorithm for MAGDM with aggregation operators}

To solve the MAGDM problem, a flowchart to solve the problem is given (see Fig. 2), the algorithm is designed as follows:

Step 1. Defining LICFV decision matrix. $\hat{D}^{t}=\left(h_{i j}^{t}\right)_{m \times k},(t=1, \ldots, n)$ is obtained by normalizing the original decision matrix $D$ in terms of Eq. (35), and we also need to check the rationality of the data.

$$
h_{i j}^{t}=\left\{\left\{\begin{array}{l}
\left.\left.\left[s_{\mu_{i j}^{-}}, s_{\mu_{i j}^{+}}\right], s_{t_{i j}}\right),\left(\left[s_{v_{i j}^{-}}, s_{v_{i j}^{+}}\right], s_{r_{i j}}\right)\right\}, \text { if the criteria is benefit type, } \\
\left.\left(\left(\left[s_{v_{i j}^{-}}, s_{v_{i j}^{+}}\right], s_{r_{i j}}\right),\left[s_{\mu_{i j}^{-}}, s_{\mu_{i j}^{+}}\right], s_{t_{i j}}\right)\right\}, \text { if the criteria is cost type. }
\end{array}\right.\right.
$$

Step 2. Aggregating all DMs. By the use of the proposed AOs to compute the LICFVs $h_{i j}^{t}(t=1, \ldots, n)$, the decision matrix of alternatives and criteria can be obtained, as $\alpha=\left(\alpha_{i j}\right)_{m \times k}$.

Step 3. Aggregating all criteria. By the used of the proposed AOs to compute the LICFVs $\alpha_{i j}(i=1, \ldots, m)$, the vectors of the alternatives are given, as $\beta=\left\{\beta_{1}, \ldots, \beta_{k}\right\}$.

Step 4. Calculating score. By the use of Eqs. (5) and (6), we compute the scores $\operatorname{Sc}\left(\beta_{j}\right)$ of all the values $\beta_{j}$. Step 5. Giving rank to the alternatives, and selecting the best one. 


\section{Case analysis}

In this section, LICFCWA and LICFCWG AOs are adopted to solve MAGDM problem. The detailed calculation steps are given, and the influence of the parameters in the AOs on the decision is deeply analyzed.

\subsection{MAGDM problem}

In this section, AOs is used to solve online education live platform selection, which is a MAGDM problem. Let four criteria $Y=\left\{Y_{1}, \ldots, Y_{4}\right\}$ and four alternatives $B=\left\{B_{1}, \ldots, B_{4}\right\}$, and whose weights are $w=$ $\left(w_{1}, \ldots, w_{4}\right)^{T}$, and $w_{i} \in[0,1], \sum_{i=1}^{4} w_{i}=1$. In addition, let three DMs $D=\left\{D^{1}, D^{2}, D^{3}\right\}$, we determine the weight of the DMs as $\lambda=\left(\lambda_{1}, \lambda_{2}, \lambda_{3}\right)^{T}$, and $\lambda_{i} \in[0,1], \sum_{i=1}^{3} \lambda_{i}=1$.

At the beginning of 2020, due to the rapid spread of COVID-19, all kinds of schools in China are delayed to open, and students are required to study at home through online education platform. In order to achieve the goal of "Stop class, but not study", teachers across the country have carried out vigorous online live teaching through QQ $\left(B_{1}\right)$, Tal's Live Online $\left(B_{2}\right)$, DingTalk $\left(B_{3}\right)$ and other platforms $\left(B_{4}\right)$. However, when evaluating the live broadcast platform, several factors are taken into consideration, such as: convenience (simple operation, $Y_{1}$ ), interactivity (in the live classroom, teachers and students can interact easily, $Y_{2}$ ), real time (in the live broadcast process, the interaction between teachers and students is very fast, $Y_{3}$ ), stability (when many people use the platform at the same time, the platform is still very stable, $Y_{4}$ ). We invited a team of teaching experts to evaluate several online platforms. To fully express the opinions of experts, they can choose from a set of predefined LTS $\mathrm{S}=\left(s_{0}\right.$ : extremely bad; $s_{1}$ : very bad; $s_{2}$ : bad; $s_{3}$ : relatively bad; $s_{4}$ : fair; $s_{5}$ : relatively good; $s_{6}$ : good; $s_{7}$ : very good; $s_{8}$ : extremely good). In addition, experts can give their preferences and non preferences for different platforms. For calculation convenience, we take the weight vector of the criterion as $w=(0.4,0.25,0.2,0.15)^{T}$ and the associated weight vector of the DMs as $\lambda=(0.243,0.514,0.243)^{T}$ based on normal distribution method (Xu 2005). LICFVs are listed in Tables 1, 2 and 3.

\subsection{The determination of the best online education platform}

In this part, some special ECs and ECCs are selected according to different generators, and the optimal platform by different methods are made.

(Case 1) We use $\mathrm{LICFC}_{\mathrm{G}} \mathrm{WA}$ and $\mathrm{LICFC}_{\mathrm{G}} \mathrm{WG}$ operators to solve this MAGMD problems, The calculation steps will be given by LICFC $_{\mathrm{G}}$ WA operators as follows:

(1) The decision matrix is obtained and preprocessed. The data in the decision matrix are all of the same type and do not need to be processed in Tables 1,2 and 3 .

(2) With LICFC $\mathrm{G}_{\mathrm{G}}$ WA operator in Eq. (21) or (33), $\theta=1.5,(\theta \geq 1)$ and $\lambda=(0.243,0.514,0.243)^{T}$ are given. Based on the aggregation of experts, LICFVs of criteria and alternatives are given (see Table 4).

(3) With LICFC $_{\mathrm{G}}$ WA operator in Eq. (21), $w=(0.2,0.3,0.25,0.25)^{T}$ is given. We aggregate the criteria to get alternatives, as shown in Table 5.

(4) With Eq. (5), we can get the scores $\operatorname{Sc}\left(B_{i}\right),(i=1,2,3,4)$, as follows:

$$
\operatorname{Sc}\left(B_{1}\right)=0.1718, \operatorname{Sc}\left(B_{2}\right)=0.0097, \operatorname{Sc}\left(B_{3}\right)=0.1471, \operatorname{Sc}\left(B_{4}\right)=0.1309 .
$$

(5) According to Definition 8, the ranking result of the evaluation can be: $B_{1}>B_{3}>B_{4}>B_{2}$.

Similarly, the ranking result of the evaluation can be $B_{1}>B_{3}>B_{4}>B_{2}$ by LICFC $_{\mathrm{G}}$ WG operators. In the calculation process of Tables 4 and 5 , we show detailed calculations given a $\theta$. Next, we analyze the influence of parameter $\theta$ on the decision result. From Fig. 3, we can know:

(1) Both the $\mathrm{LICFC}_{\mathrm{G}} \mathrm{WA}$ and $\mathrm{LICFC}_{\mathrm{G}} \mathrm{WG}$ give the same optimal decision $B_{1}$. This shows that the designed AOs are effective.

(2) The parameter $\theta$ has some influence on the ordering of other alternatives. The specific results are as follows:

(a) When $\theta \in[-1,0) \bigcup(0,1.2677]$, the sorting result is $B_{1}>B_{3}>B_{4}>B_{2}$ by LICFC $_{\mathrm{G}} \mathrm{WA}$, when $\theta \in[1.2678,10]$, the sorting result is $B_{1}>B_{4}>B_{3}>B_{2}$ by LICFC $_{\mathrm{G}}$ WA.

(b) When $\theta \in[-1,0) \cup(0,5.9275]$, the sorting result is $B_{1}>B_{3}>B_{4}>B_{2}$ by $\mathrm{LICFC}_{\mathrm{G}} \mathrm{WG}$, when $\theta \in[5.9276,10]$, the sorting result is $B_{1}>B_{3}>B_{2}>B_{4}$ by LICFC $_{\mathrm{G}}$ WA. 


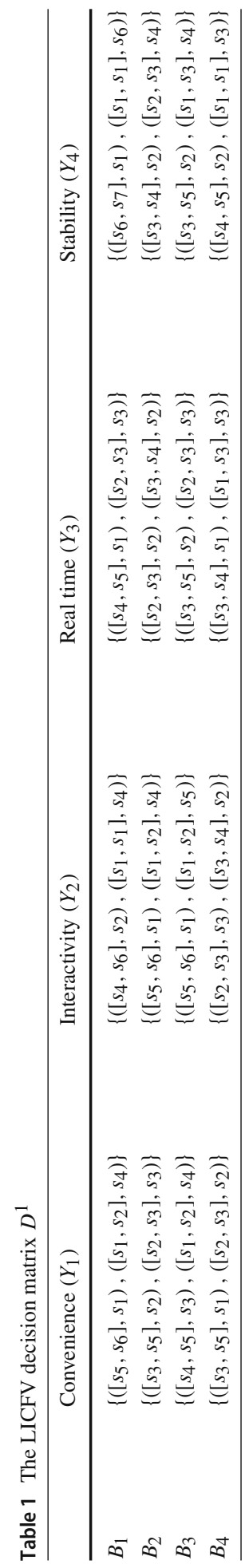




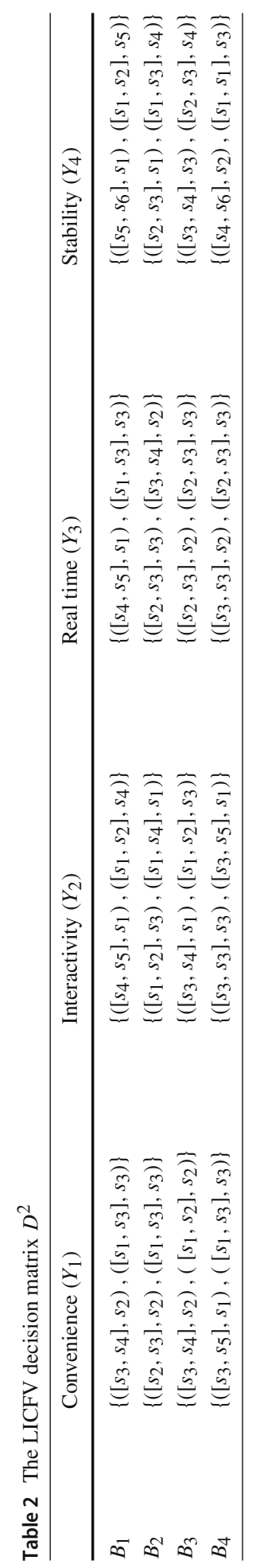




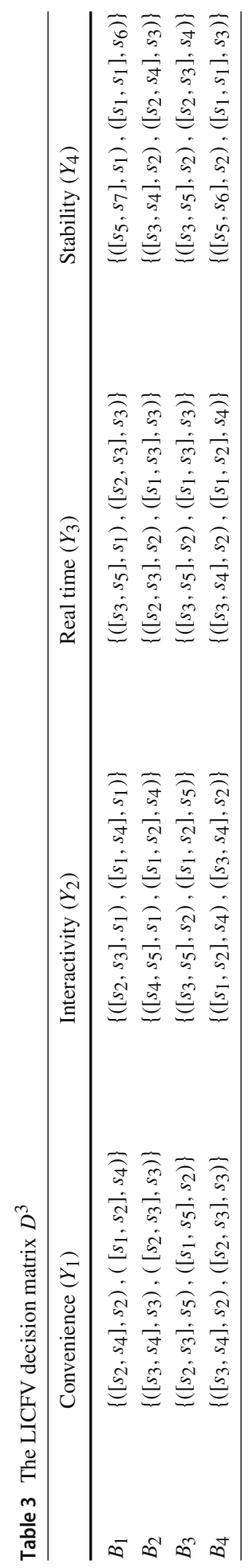




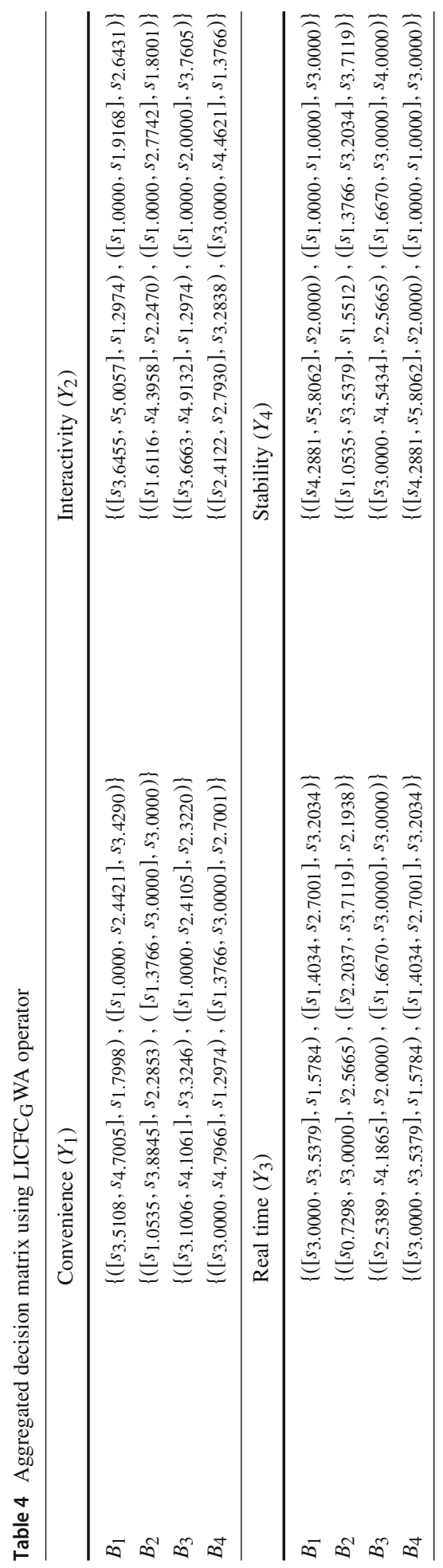


Table 5 Aggregated alternatives using LICFC $_{\mathrm{G}}$ WA operator

\begin{tabular}{ll}
\hline$B_{1}$ & $\left\{\left(\left[s_{3.9677}, s_{5.2715}\right], s_{1.4276}\right),\left(\left[s_{1.0638}, s_{2.1796}\right], s_{3.2992}\right)\right\}$ \\
$B_{2}$ & $\left\{\left(\left[s_{1.1574}, s_{3.8308}\right], s_{2.2415}\right),\left(\left[s_{1.3822}, s_{3.0918}\right], s_{2.5236}\right)\right\}$ \\
$B_{3}$ & $\left\{\left(\left[s_{3.1445}, s_{4.4161}\right], s_{2.5805}\right),\left(\left[s_{1.1864}, s_{2.4707}\right], s_{2.9526}\right)\right\}$ \\
$B_{4}$ & $\left\{\left(\left[s_{3.1223}, s_{4.4452}\right], s_{2.1110}\right),\left(\left[s_{1.5636}, s_{2.6252}\right], s_{2.3481}\right)\right\}$ \\
\hline
\end{tabular}
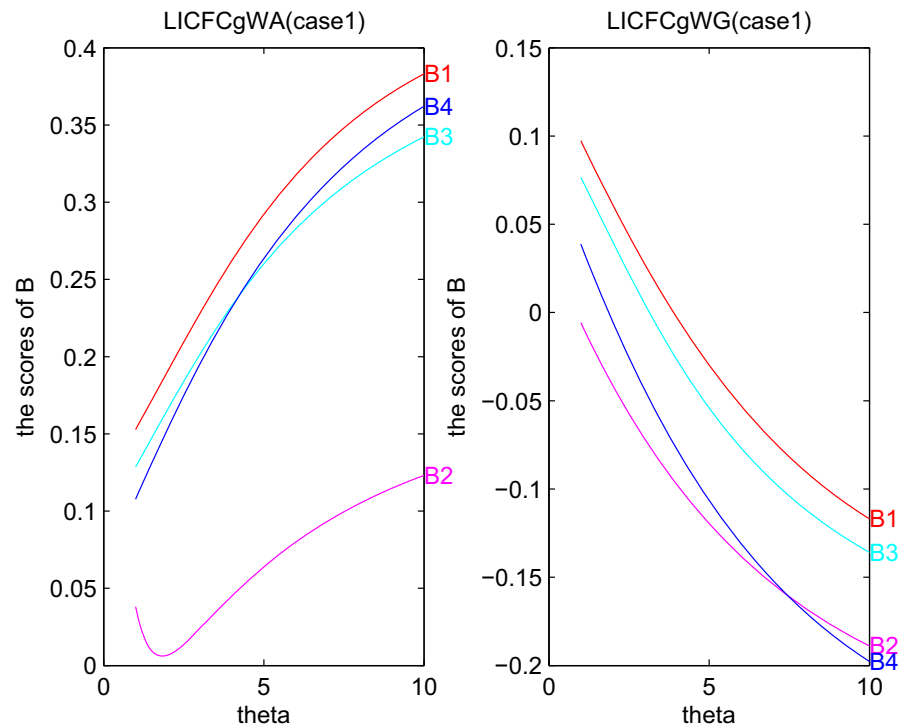

Fig. 3 Scores of $B_{i}(i=1,2,3,4)$ when $\theta \in[1,10]$

(3) The score values of the four alternatives increase with the increase of $\theta$ by LICFC $_{\mathrm{G}} \mathrm{WA}$, but the score values of the four alternatives decrease with the increase of $\theta$ by LICFC $_{\mathrm{G}}$ WG. We recommend that the value of the parameter be as small as possible. For example, the value range of parameter $\theta \in[1,2]$.

(Case 2) We use ICFC $_{C}$ WA and ICFC $_{C}$ WG operators to solve this MAGDM problem. Similar to Case 1, we can get the result, as shown in Fig. 4. From Fig. 4, we can know:

(1) Both the $\mathrm{LICFC}_{\mathrm{C}} \mathrm{WA}$ and $\mathrm{LICFC}_{\mathrm{C}} \mathrm{WG}$ give the same optimal decision $B_{1}$. This shows that the designed AOs are effective.

(2) The parameter $\theta$ has some influence on the ordering of other alternatives. The specific results are as follows:

(a) When $\theta \in[-1,0) \bigcup(0,1.2677]$, the sorting result is $B_{1}>B_{3}>B_{4}>B_{2}$ by LICFC $\mathrm{CWA}$, when $\theta \in[1.2678,10]$, the sorting result is $B_{1}>B_{4}>B_{3}>B_{2}$ by LICFC $C$ WA.

(b) When $\theta \in[-1,0) \cup(0,5.9275]$, the sorting result is $B_{1}>B_{3}>B_{4}>B_{2}$ by LICFC $\mathrm{CWG}$, when $\theta \in[5.9276,10]$, the sorting result is $B_{1}>B_{3}>B_{2}>B_{4}$ by LICFC $_{C}$ WA.

(3) The score values of the four alternatives increase with the increase of $\theta$ by LICFC $C$ WA, but the score values of the four alternatives decrease with the increase of $\theta$ by LICFC $_{C} \mathrm{WG}$, We recommend that the value of the parameter be as small as possible. For example, the value range of parameter $\theta \in[-1,0) \bigcup(0,1]$.

(Case 3) We use LICFC $_{F}$ WA and LICFC $_{F}$ WG operators to solve this MAGDM problem. Similar to Case 1, we can get the result, as shown in Fig. 5. We can know from Fig. 5:

(1) Whether we use LICFC $_{\mathrm{F}} \mathrm{WA}$ or $\mathrm{LICFC}_{\mathrm{F}} \mathrm{WG}$, we can get the same sorting result: $B_{1}>B_{3}>B_{2}>B_{4}$.

(2) The score values of the four alternatives increase with the increase of $\theta$ by LICFC $_{F} W A$, but the score values of the four alternatives decrease with the increase of $\theta$ by LICFC $_{\mathrm{F}} \mathrm{WG}$.

In addition, the value of parameter $\theta$ should be as small as possible. We suggest $\theta \in[-1,0) \bigcup(0,1]$. 

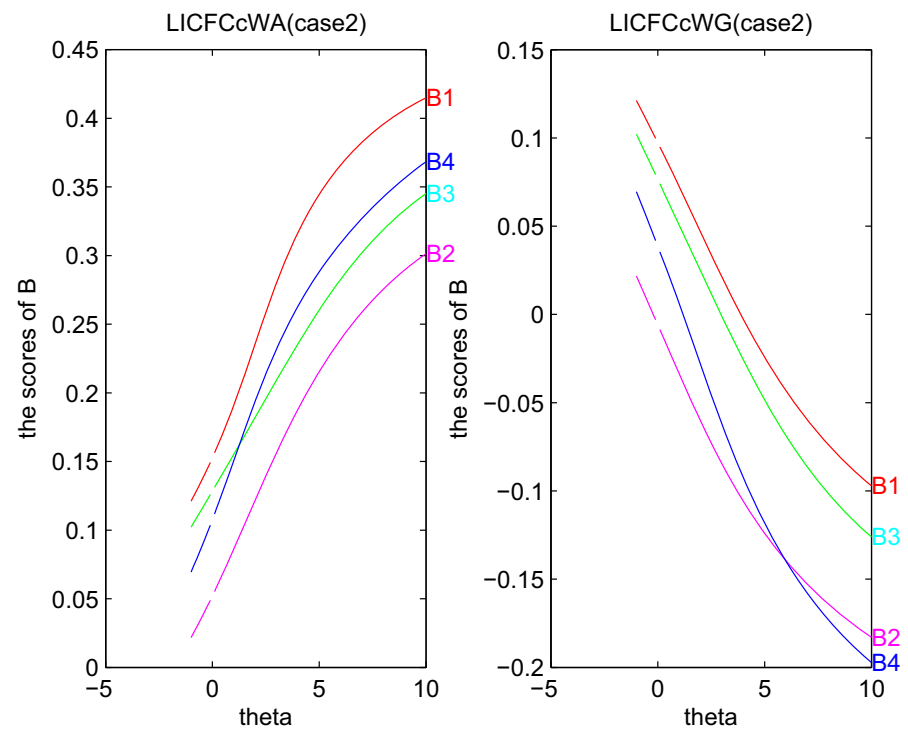

Fig. 4 Scores of $B_{i}(i=1,2,3,4)$ when $-1 \leq \theta \leq 10$ and $\theta \neq 0$
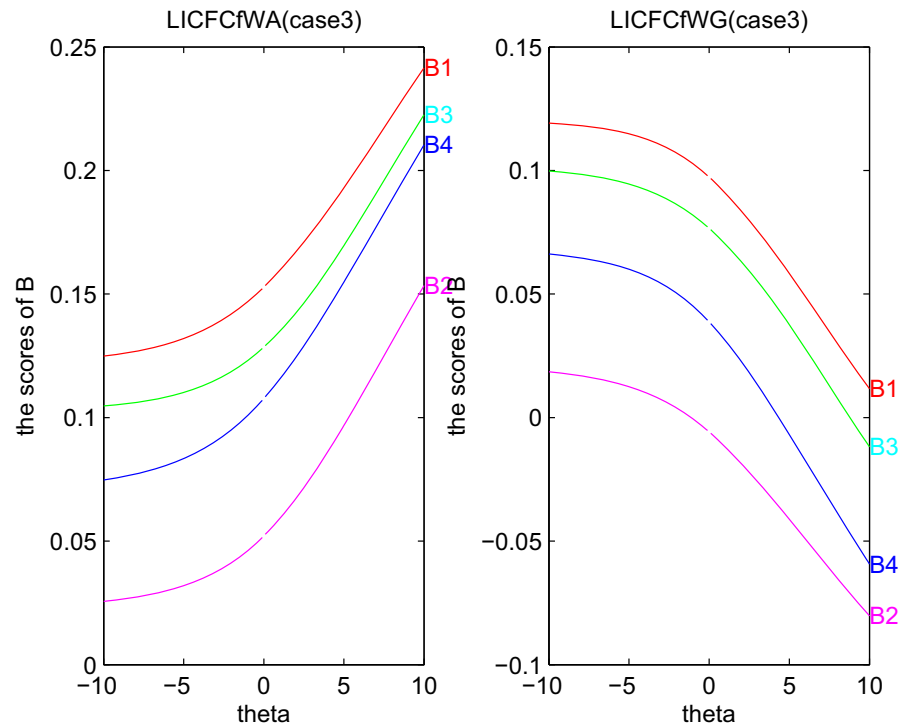

Fig. 5 Scores of $B_{i}(i=1,2,3,4)$ when $\theta \neq 0$, and $\theta \in[-10,10]$

(Case 4) We use LICFC $_{A}$ WA and LICFC $_{A}$ WG operators to solve this MAGDM problems. Similar to Case 1, we can get the result, as shown in Fig. 6.

From Fig. 6, The conclusion is the same as that in case 3, the ranking result of the evaluation can be: $B_{1}>B_{3}>B_{4}>B_{2}$ by LICFC $_{\mathrm{A}} \mathrm{WA}$ or $\mathrm{LICFC}_{\mathrm{A}} \mathrm{WG}$. In addition, we suggest that the value of parameter is $[-1,1)$.

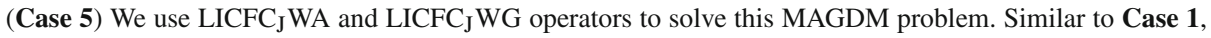
we can get the result, as shown in Fig. 7. 

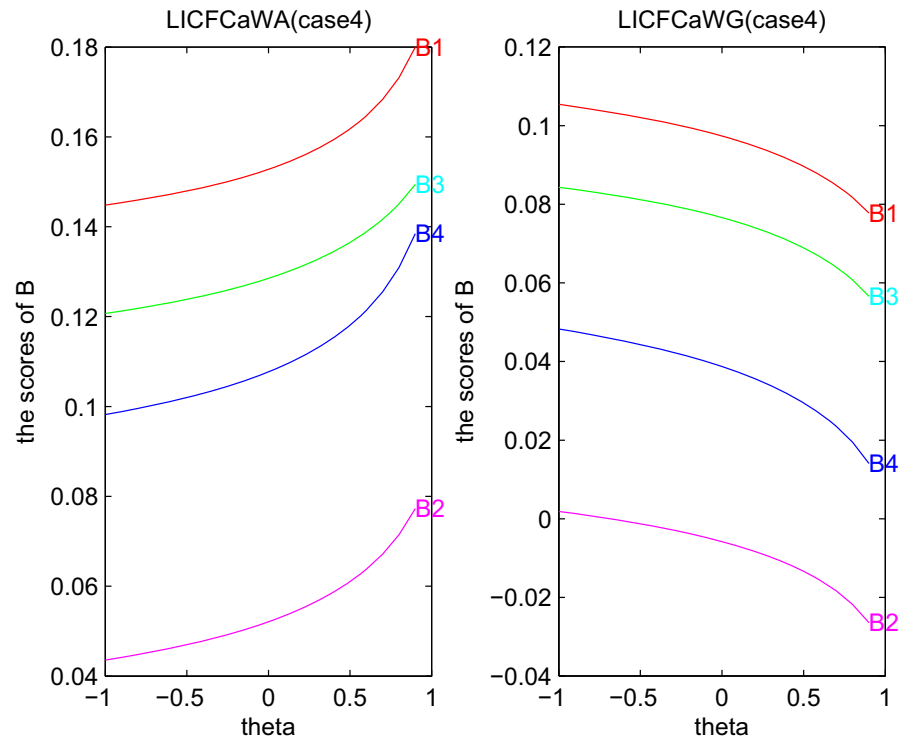

Fig. 6 Scores of $B_{i}(i=1,2,3,4)$ when $\theta \in[-1,1)$

(1) Both the $\mathrm{LICFC}_{\mathrm{J}} \mathrm{WA}$ and $\mathrm{LICFC}_{\mathrm{J}} \mathrm{WG}$ give the same optimal decision $B_{1}$. This shows that the designed AOs are effective.

(2) The parameter $\theta$ has some influence on the ordering of other alternatives. The specific results are as follows:

(a) When $\theta \in[1,15.9249]$, the sorting result is $B_{1}>B_{3}>B_{4}>B_{2}$ by LICFC $\mathrm{J}$ WA, when $\theta \in$ [15.9250, 20], the sorting result is $B_{1}>B_{4}>B_{3}>B_{2}$ by LICFC $_{J}$ WA.

(b) When $\theta \in[1,14.0345]$, the sorting result is $B_{1}>B_{3}>B_{4}>B_{2}$ by LICFC JWG, when $\theta \in$ [14.0346, 20], the sorting result is $B_{1}>B_{3}>B_{2}>B_{4}$ by LICFC ${ }_{\mathrm{J}}$ WG.

(3) The score values of the four alternatives increase with the increase of $\theta$ by LICFCJWA, but the score

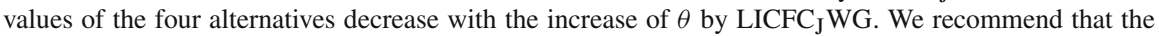
value of the parameter be as small as possible. We suggest that the value range of the parameter is $[1,3]$.

From the above case study, we know:

(1) If we uniformly reduce the value range of the parameter to a smaller interval within the definition domain, for example, $[-1,0],[0,1]$ or $[1,2]$, we can get the same sorting result: $B_{1}>B_{3}>B_{4}>B_{2}$.

(2) In different AOs, the value of the score function calculated by the algebraic average operator increases with the increase of the parameters, while the value of the score function calculated by the geometric average operator is just the opposite.

(3) The proposed AOs is effective.

\section{Comparison analysis with other methods}

In this section, first, the experimental data of this paper were used to compare the methods of this paper with LICFWA, LICFOWA, LICFHA, LICFWG, LICFOWG and LICFHG (Qiyas et al. 2020). Secondly, LICFS is reduced to LIFS and ICFS, respectively. In the case of the same experimental data and the parameters, the validity and flexibility of the proposed approaches are verified by comparing it with the existing MAGDM approaches. 

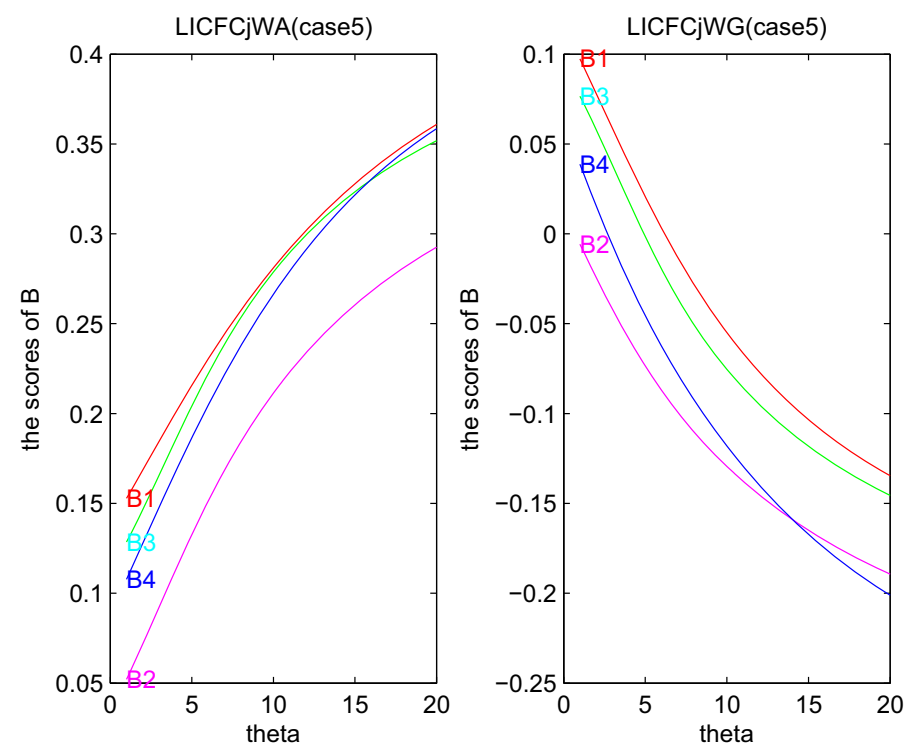

Fig. 7 Scores of $B_{i}(i=1,2,3,4)$ when $\theta \in[1,20]$

\subsection{Comparative experiment 1}

Our methods are compared with the methods in reference (Qiyas et al. 2020) to illustrate the effectiveness and advantages of our methods. To make the comparison more reasonable, we take the weight vector of the criterion as $w=(0.4,0.25,0.2,0.15)^{T}$ and the associated weight vector of the DMs as $\lambda=(0.243,0.514,0.243)^{T}$. LICFVs are listed in Tables 1, 2 and 3. The score function defined in this paper is used in the comparison experiments. The comparison results are shown in Table 6.

From Table 6, it can be seen that for the same problems and parameters, the optimal decision given in reference (Qiyas et al. 2020) is consistent with the results obtained by the method in this paper. By comparing the two types of methods (the methods in this paper and the methods in reference Qiyas et al. 2020), we can reach the following conclusions: First, for the same practical problem, both types of methods can give the optimal decision $B_{1}$. This shows that both types of methods are valid. Second, the methods in reference (Qiyas et al. 2020) are not flexible enough to change the method according to the characteristics of the problem or the type of experts, while the methods in this paper can choose different types of functions and have more flexible parameter adjustment functions.

\subsection{Comparative experiment 2}

In this part, the AOs of this paper is compared with the existing fuzzy AOs, and draw a conclusion. In the practical problems, there are some problems that interval-valued linguistic intuitionistic fuzzy set can not solve, but this paper proposes a broader structure, which can solve more practical problems, which also shows the limitations of the existing methods. In order to compare with other existing methods, we can deal with the examples in other papers: convert the data of interval-valued to mean value or rewrite the value to intervalvalued, and assign the value other than interval-valued to zero, so as to compare the data of different problems. If we deal with any problem under the linguistic intuitionistic fuzzy information, we can easily use LICFS to solve the problem by converting the linguistic intuitionistic fuzzy variable (LIFV) into the LICFV. We can set all the non-interval-valued of the linguistic intuitionistic fuzzy number to be equal. In order to be simplified and to satisfy some of the conditions of a linguistic intuitionistic cubic fuzzy set, we can set it to zero, i.e., the LIFV ([s,$\left.\left.s_{3}\right],\left[s_{3}, s_{4}\right]\right)$ can be converted to LICFV $\left\{\left(\left[s_{2}, s_{3}\right], s_{0}\right),\left(\left[s_{3}, s_{4}\right], s_{0}\right)\right\}$. Next, we compare this method with Liu and Wang (2017), Garg and Kumar (2018, 2019), Liu and Qin (2017) and Liu and Liu (2017). In order to compare the rationality, we use the same data (see Table 6) and parameters. In all AOs, the attribute weight vector is $w=(0.4,0.25,0.2,0.15)^{T}$, the expert weight vector is $\lambda=(0.243,0.514,0.243)^{T}$. 


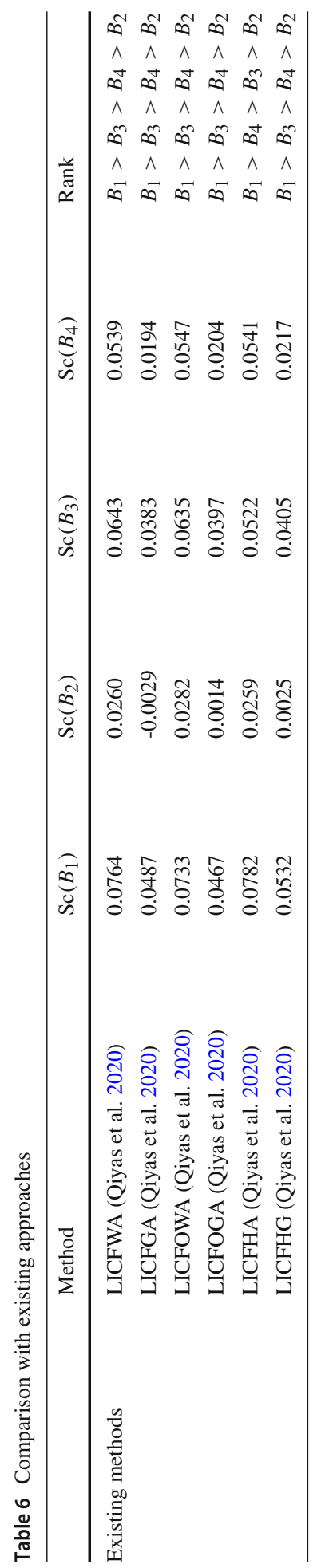


Table 7 Decision matrix $D^{k}(k=1,2,3)$ (Garg and Kumar 2019)

\begin{tabular}{|c|c|c|c|c|c|}
\hline & & $Y_{1}$ & $Y_{2}$ & $Y_{3}$ & $Y_{4}$ \\
\hline \multirow[t]{4}{*}{$D^{1}$} & $B_{1}$ & $\left(\left[s_{5}, s_{6}\right],\left[s_{1}, s_{2}\right]\right)$ & $\left(\left[s_{4}, s_{6}\right],\left[s_{1}, s_{1}\right]\right)$ & $\left(\left[s_{4}, s_{5}\right],\left[s_{2}, s_{3}\right]\right)$ & $\left(\left[s_{6}, s_{7}\right],\left[s_{1}, s_{1}\right]\right)$ \\
\hline & $B_{2}$ & $\left(\left[s_{3}, s_{5}\right],\left[s_{2}, s_{3}\right]\right)$ & $\left(\left[s_{5}, s_{6}\right],\left[s_{1}, s_{2}\right]\right)$ & $\left(\left[s_{2}, s_{4}\right],\left[s_{3}, s_{4}\right]\right)$ & $\left(\left[s_{3}, s_{4}\right],\left[s_{2}, s_{3}\right]\right)$ \\
\hline & $B_{3}$ & $\left(\left[s_{5}, s_{6}\right],\left[s_{1}, s_{2}\right]\right)$ & $\left(\left[s_{5}, s_{6}\right],\left[s_{1}, s_{2}\right]\right)$ & $\left(\left[s_{3}, s_{5}\right],\left[s_{2}, s_{3}\right]\right)$ & $\left(\left[s_{3}, s_{5}\right],\left[s_{1}, s_{3}\right]\right)$ \\
\hline & $B_{4}$ & $\left(\left[s_{4}, s_{5}\right],\left[s_{2}, s_{3}\right]\right)$ & $\left(\left[s_{1}, s_{3}\right],\left[s_{3}, s_{4}\right]\right)$ & $\left(\left[s_{3}, s_{5}\right],\left[s_{1}, s_{3}\right]\right)$ & $\left(\left[s_{6}, s_{7}\right],\left[s_{1}, s_{1}\right]\right)$ \\
\hline \multirow[t]{4}{*}{$D^{2}$} & $B_{1}$ & $\left(\left[s_{2}, s_{4}\right],\left[s_{1}, s_{3}\right]\right)$ & $\left(\left[s_{4}, s_{5}\right],\left[s_{1}, s_{2}\right]\right)$ & $\left(\left[s_{4}, s_{5}\right],\left[s_{1}, s_{3}\right]\right)$ & $\left(\left[s_{3}, s_{6}\right],\left[s_{1}, s_{2}\right]\right)$ \\
\hline & $B_{2}$ & $\left(\left[s_{3}, s_{5}\right],\left[s_{1}, s_{3}\right]\right)$ & $\left(\left[s_{1}, s_{2}\right],\left[s_{1}, s_{4}\right]\right)$ & $\left(\left[s_{2}, s_{3}\right],\left[s_{3}, s_{4}\right]\right)$ & $\left(\left[s_{3}, s_{5}\right],\left[s_{1}, s_{3}\right]\right)$ \\
\hline & $B_{3}$ & $\left(\left[s_{3}, s_{4}\right],\left[s_{1}, s_{2}\right]\right)$ & $\left(\left[s_{3}, s_{6}\right],\left[s_{1}, s_{2}\right]\right)$ & $\left(\left[s_{2}, s_{5}\right],\left[s_{2}, s_{3}\right]\right)$ & $\left(\left[s_{3}, s_{4}\right],\left[s_{2}, s_{3}\right]\right)$ \\
\hline & $B_{4}$ & $\left(\left[s_{4}, s_{5}\right],\left[s_{1}, s_{2}\right]\right)$ & $\left(\left[s_{3}, s_{3}\right],\left[s_{3}, s_{5}\right]\right)$ & $\left(\left[s_{3}, s_{3}\right],\left[s_{2}, s_{3}\right]\right)$ & $\left(\left[s_{4}, s_{6}\right],\left[s_{1}, s_{1}\right]\right)$ \\
\hline \multirow[t]{4}{*}{$D^{3}$} & $B_{1}$ & $\left(\left[s_{2}, s_{4}\right],\left[s_{1}, s_{2}\right]\right)$ & $\left(\left[s_{2}, s_{3}\right],\left[s_{1}, s_{4}\right]\right)$ & $\left(\left[s_{3}, s_{5}\right],\left[s_{2}, s_{3}\right]\right)$ & $\left(\left[s_{5}, s_{7}\right],\left[s_{1}, s_{1}\right]\right)$ \\
\hline & $B_{2}$ & $\left(\left[s_{1}, s_{4}\right],\left[s_{2}, s_{3}\right]\right)$ & $\left(\left[s_{4}, s_{5}\right],\left[s_{1}, s_{2}\right]\right)$ & $\left(\left[s_{2}, s_{4}\right],\left[s_{1}, s_{3}\right]\right)$ & $\left(\left[s_{3}, s_{4}\right],\left[s_{2}, s_{4}\right]\right)$ \\
\hline & $B_{3}$ & $\left(\left[s_{2}, s_{3}\right],\left[s_{1}, s_{5}\right]\right)$ & $\left(\left[s_{3}, s_{5}\right],\left[s_{1}, s_{2}\right]\right)$ & $\left(\left[s_{3}, s_{5}\right],\left[s_{1}, s_{3}\right]\right)$ & $\left(\left[s_{3}, s_{5}\right],\left[s_{2}, s_{3}\right]\right)$ \\
\hline & $B_{4}$ & $\left(\left[s_{3}, s_{4}\right],\left[s_{2}, s_{3}\right]\right)$ & $\left(\left[s_{1}, s_{2}\right],\left[s_{3}, s_{4}\right]\right)$ & $\left(\left[s_{3}, s_{5}\right],\left[s_{1}, s_{2}\right]\right)$ & $\left(\left[s_{5}, s_{6}\right],\left[s_{1}, s_{1}\right]\right)$ \\
\hline
\end{tabular}

Table 8 Comparison with existing approaches

\begin{tabular}{|c|c|c|c|c|c|c|}
\hline & Method & $\operatorname{Sc}\left(B_{1}\right)$ & $\operatorname{Sc}\left(B_{2}\right)$ & $\operatorname{Sc}\left(B_{3}\right)$ & $\operatorname{Sc}\left(B_{4}\right)$ & Rank \\
\hline \multirow[t]{5}{*}{ Existing methods } & Liu and Wang (2017) & 2.5259 & 1.0904 & 2.0827 & 1.7157 & $B_{1}>B_{3}>B_{4}>B_{2}$ \\
\hline & Garg and Kumar (2018) & 4.5958 & 4.3111 & 4.5058 & 4.3264 & $B_{1}>B_{3}>B_{4}>B_{2}$ \\
\hline & Liu and Qin (2017) & -6.6078 & -6.9589 & -6.7591 & -6.8626 & $B_{1}>B_{3}>B_{4}>B_{2}$ \\
\hline & Liu and Liu (2017) & 6.5503 & 6.3528 & 6.5002 & 6.4590 & $B_{1}>B_{3}>B_{4}>B_{2}$ \\
\hline & Garg and Kumar (2019) & 5.4172 & 4.7036 & 5.1332 & 4.9454 & $B_{1}>B_{3}>B_{4}>B_{2}$ \\
\hline \multirow[t]{10}{*}{ Proposed methods } & LICFC $_{\mathrm{G}}$ WA (Case 1) & 5.4259 & 4.3031 & 5.1716 & 5.0816 & $B_{1}>B_{3}>B_{4}>B_{2}$ \\
\hline & LICFC $_{C}$ WA (Case 2) & 5.5917 & 4.7857 & 5.2622 & 5.3139 & $B_{1}>B_{3}>B_{2}>B_{4}$ \\
\hline & LICFC $_{F}$ WA (Case 3) & 4.9785 & 4.3780 & 4.9613 & 4.7187 & $B_{1}>B_{3}>B_{4}>B_{2}$ \\
\hline & LICFC $_{A}$ WA (Case 4) & 4.9705 & 4.3661 & 4.9476 & 4.7083 & $B_{1}>B_{3}>B_{4}>B_{2}$ \\
\hline & $\mathrm{LICFC}_{\mathrm{J}} \mathrm{WA}($ Case 5) & 4.9624 & 4.3697 & 4.9507 & 4.7052 & $B_{1}>B_{3}>B_{4}>B_{2}$ \\
\hline & LICFC $_{G}$ WG (Case 1) & 5.0263 & 4.2628 & 4.8445 & 4.4454 & $B_{1}>B_{3}>B_{4}>B_{2}$ \\
\hline & LICFC $_{C}$ WG (Case 2) & 4.9645 & 4.1334 & 4.7874 & 4.2730 & $B_{1}>B_{3}>B_{4}>B_{2}$ \\
\hline & LICFC $_{F}$ WG (Case 3) & 4.5251 & 3.9097 & 4.4602 & 4.1627 & $B_{1}>B_{3}>B_{4}>B_{2}$ \\
\hline & LICFC $_{A}$ WG (Case 4) & 4.5380 & 3.9199 & 4.4727 & 4.1770 & $B_{1}>B_{3}>B_{4}>B_{2}$ \\
\hline & $\mathrm{LICFC}_{\mathrm{J}} \mathrm{WG}$ (Case 5) & 4.5276 & 3.9114 & 4.4692 & 4.1635 & $B_{1}>B_{3}>B_{4}>B_{2}$ \\
\hline
\end{tabular}

According to the parameter value range in ECs and ECCs, $\theta=0.5$ in Case 4, and $\theta=1.5$ for other Cases. In addition, we take the score function in Garg and Kumar (2019), namely

$$
\operatorname{Sc}(h)=\frac{2 \ell+\mu^{-}+\mu^{+}-v^{-}-v^{+}}{4} .
$$

The comparison results are shown in Table 8 .

We can see from Table 8 that for the same decision-making problem (Table 7), the AOs in this paper give the best choice with the same parameters: $B_{1}$, the ranking results of other alternatives are almost the same, only the worst alternatives in $\mathrm{LICFC}_{\mathrm{C}} \mathrm{WA}$ (Case 2) give different results. In addition, for the decisionmaking problem in this section, we arbitrarily choose Case $\mathbf{4}$ as the aggregation function and carry out detailed parameter analysis. The results are shown in Fig. 8. We can see that the aggregation method in this paper has good adaptability and can solve the decision-making problems in different fuzzy environments. Using other 

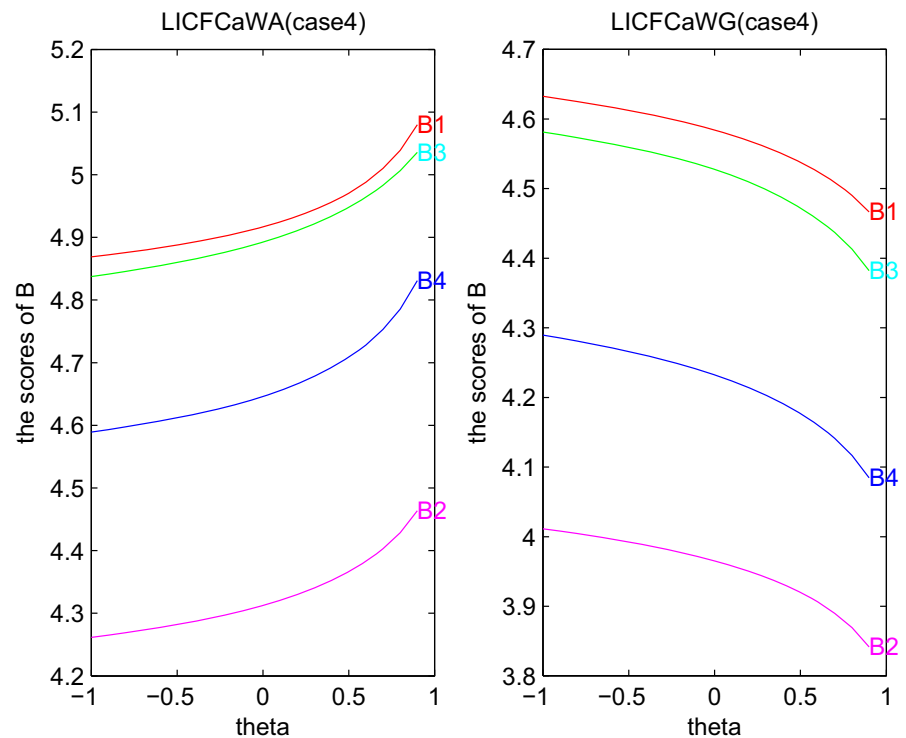

Fig. 8 Scores of $B_{i}(i=1,2,3,4)$ when $\theta \in[-1,1)$

aggregate functions, we can also get similar parametric analysis graphs. Due to the limited space, no other graphs are given.

\subsection{Comparative experiment 3}

In this section, the method of this paper are used to deal with the problem of MAGDM in intuitionistic cubic fuzzy environment. First of all, we transform the LICFS into intuitionistic cubic fuzzy set (ICFS) by the following ways.

$$
h^{\prime}=\left\{\left\langle\left[\frac{\mu^{-}}{\ell}, \frac{\mu^{+}}{\ell}\right], \frac{\phi}{\ell}\right\rangle,\left\langle\left[\frac{v^{-}}{\ell}, \frac{v^{+}}{\ell}\right], \frac{\varphi}{\ell}\right\rangle\right\}=\left\{\left\langle\left[e^{-}, e^{+}\right], p\right\rangle,\left\langle\left[r^{-}, r^{+}\right], q\right\rangle\right\} .
$$

In addition, to make the comparison reasonable, we adopt the decision matrix (see Table 9; Muneeza and Abdullah 2020) and score function in Muneeza and Abdullah (2020). The same parameters are used, the attribute $(Y)$ weight vector is $w=(0.2,0.3,0.10 .4)^{T}$, the expert weight vector is $\lambda=(0.4,0.25,0.35)^{T}$. According to the parameter value range in ECs and ECCs, $\theta=0.5$ in Case 4, and $\theta=1.5$ for other Cases. The score function (Muneeza and Abdullah 2020) is defined as follows:

$$
\operatorname{Sc}\left(h^{\prime}\right)=\frac{e^{-}+e^{+}+p-r^{-}-r^{+}-q}{4} .
$$

According to Table 10 and Fig. 9, we can get the following conclusions:

(1) The given method can solve MAGDM problems in intuitionistic cubic fuzzy environment. This shows that this method has strong adaptability and flexibility.

(2) Under different AOs, this method can give the best option $B_{1}$ and the worst option $B_{4}$. However, some of the methods listed in Muneeza and Abdullah (2020) are not stable. Therefore, the method in this paper is better than that in Muneeza and Abdullah (2020).

(3) We take Case $\mathbf{4}$ as an example to analyze the parameters in detail, which can verify the stability of this method. Only when $\theta=-0.354$, the order between alternative $B_{2}$ and alternative $B_{3}$ is adjusted by $\mathrm{LICFC}_{\mathrm{A}} \mathrm{WG}$ operator, but the optimal option is not affected. In addition, through the parameter analysis of other Cases, we can get a similar conclusion: the stability is very good. 


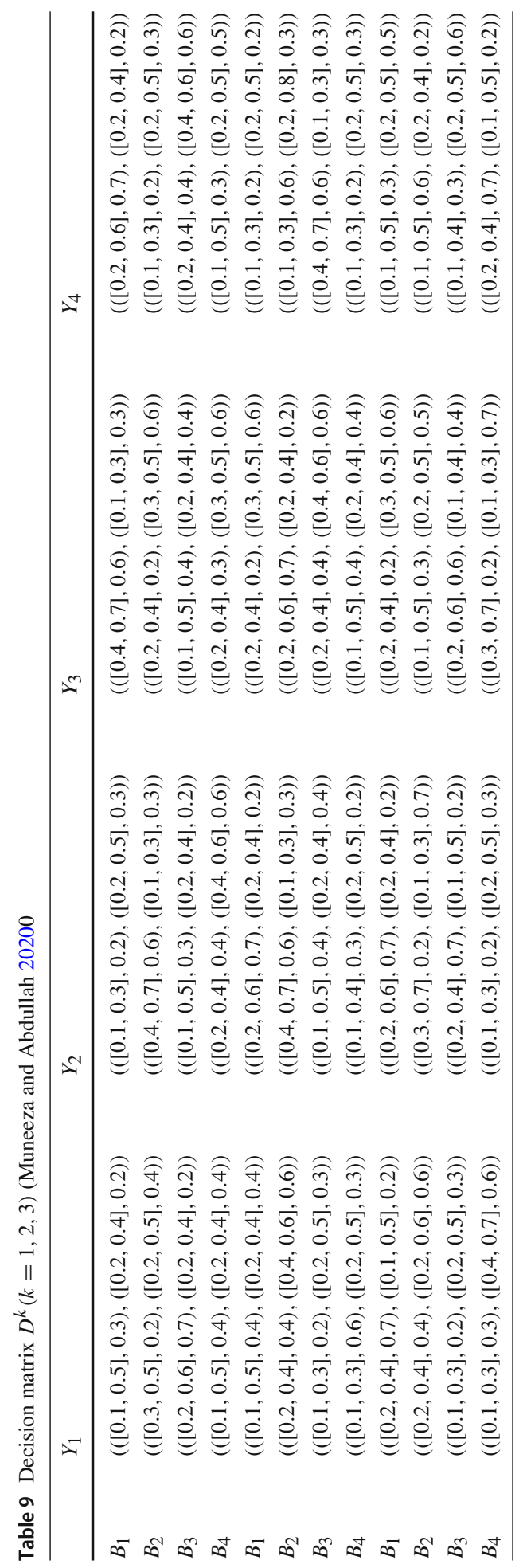




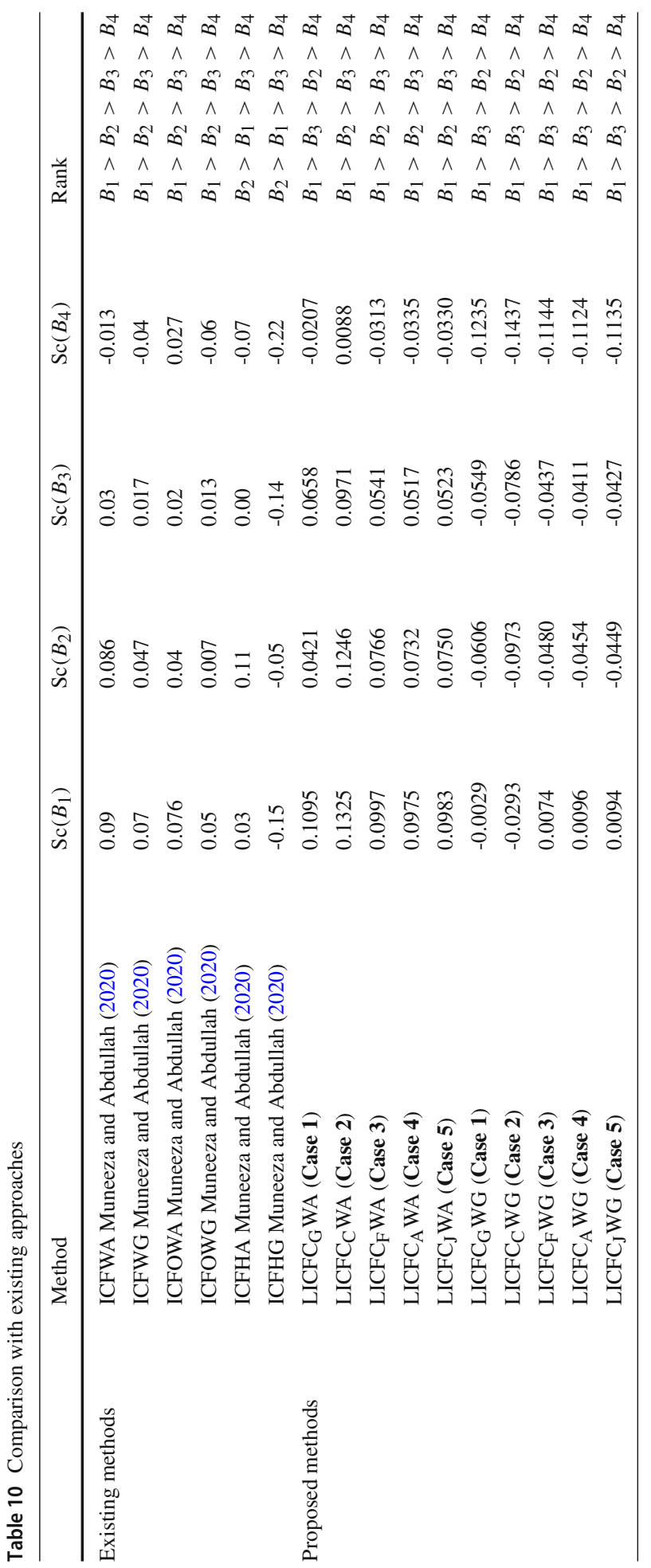



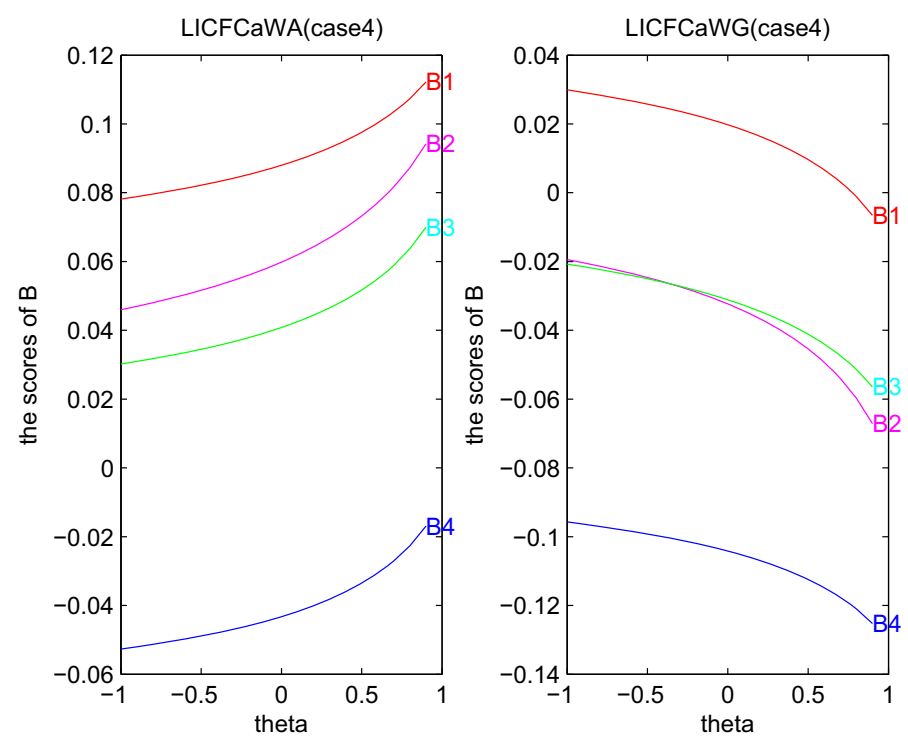

Fig. 9 Scores of $B_{i}(i=1,2,3,4)$ when $\theta \in[-1,1)$

\section{Conclusions}

The main work of this paper is to study the AOs based on extended copulas and co-copulas under linguistic intuitionistic cubic fuzzy environment. Based on specific instances of ECs and ECCs a series of AOs are constructed. These AOs have achieved good results in solving multi-attribute group decision problems. In particular, in the study of the selection of online education platforms, we conducted detailed parameter analysis of these AOs and gave the effect of parameter changes on decision results. The results show that the constructed AOs perform well. Moreover, we degenerate LICFS into LIFS and LICFS into ICFS, and conduct detailed experimental comparisons under the same conditions. The results show that our AOs can solve the MAGDM problem of LIFSs and ICFSs. This also shows that our AOs have good portability.

In multi-granularity fuzzy linguistic modeling, each expert is allowed to use his own LTS to express his preferences, and it has been widely used in the field of MAGDM. However, most of the literature is given the weight information of the experts and the weight information of the given attributes, and we know that the weight information has an extremely important influence on the aggregation results. Therefore, in future research, we will define the distance between LICFVs, and construct an optimization model to solve the weights of attributes and the weights of DMs, which can make our decision more objective.

In addition, with the continuous development of COVID-19, different online education platforms have fully reflected their advantages and disadvantages. According to the statistical survey of users and the research results of this paper, we give real-time feedback to relevant online education platforms, so that the development of online education platforms can be better utilized and users will have better experience. For similar problems, the method in this paper can make the optimal choice more reasonably.

Acknowledgements This work was supported by Sichuan Province Youth Science and Technology Innovation Team (No. 2019JDTD0015); Scientific Research Innovation Team of Neijiang Normal University (No. 18TD008); Open fund of Data Recovery Key Lab of Sichuan Province (No. DRN19018).

\section{References}

Arora R, Garg H (2019) Group decision-making method based on prioritized linguistic intuitionistic fuzzy aggregation operators and its fundamental properties. Comput Appl Math. https://doi.org/10.1007/ s40314-019-0764-1 
Atanassov K (1986) Intuitionistic fuzzy sets. Fuzzy Sets Syst 20:87-96

Atanassov K, Gargov G (1989) Interval valued intuitionistic fuzzy sets. Fuzzy Sets Syst 31:343-349

Bedregal B, Reiser R, Bustince H, Molina CL, Torra V (2014) Aggregation functions for typical hesitant fuzzy elements and the action of automorphisms. Inf Sci 255:82-99

Chen $\mathrm{Z}$ et al (2015) An approach to multiple attribute group decision making based on linguistic intuitionistic fuzzy numbers. Int J Comput Intell Syst 8:747-760

Chen T, He SS et al (2018) Novel operations for linguistic neutrosophic sets on the basis of Archimedean copulas and co-copulas and their application in multi-criteria decision-making problems. J Intell Fuzzy Syst 37:2887-2912

Chen S, Wang J, Wang T (2019) Cloud-based ERP system selection based on extended probabilistic linguistic MULTIMOORA method and Choquet integral operator. Comput Appl Math 38:2. https://doi.org/10. 1007/s40314-019-0839-z

Cuong BC, Phong PH (2015) Max-Min composition of linguistic intuitionistic fuzzy relations and application in medical diagnosis. VNU J Sci Comput Sci Commun Eng. https://doi.org/10.1143/PTP.53.1392

Fahmi A, Abdullah S, Amin F, Siddiqui N, Ali A (2017) Aggregation operators on triangular cubic fuzzy numbers and its application to multi-criteria decision making problems. J Intell Fuzzy Syst 33:33233337

Fahmi A, Abdullah S, Amin F, Siddiqui N, Ali A (2018a) Triangular cubic linguistic hesitant fuzzy aggregation operators and their application in group decision making. J Intell Fuzzy Syst 34:2401-2416

Fahmi A, Abdullah S, Amin F, Ali A, Ahmad KW (2018b) Some geometric operators with triangular cubic linguistic hesitant fuzzy number and their application in group decision-making. J Intell Fuzzy Syst. https://doi.org/10.3233/jifs-18125

Fahmi A, Amin F, Abdullah S, Ali A (2018) Cubic fuzzy Einstein aggregation operators and its application to decision-making. Int J Syst Sci. https://doi.org/10.1080/00207721.2018.1503356

Fahmi A, Abdullah S, Amin F, Khan MSA (2018d) Trapezoidal cubic fuzzy number Einstein hybrid weighted averaging operators and its application to decision making. Soft Comput. https://doi.org/10.1007/s00500018-3242-6

Fahmi A, Amin F, Abdullah S, Aslam M, Amin N (2019) Cubic Fuzzy multi-attribute group decision-making with an application to plant location selected based on a new extended Vikor method. J Intell Fuzzy Syst 37:583-596

Garg H (2018) Linguistic Pythagorean fuzzy sets and its applications in multiattribute decision-making process. Int J Intell Syst 33:1234-1263

Garg H, Kumar K (2018) Some aggregation operators for linguistic intuitionistic fuzzy set and its application to group decision-making process using the set pair analysis. Arab J Sci Eng 43:3213-3227

Garg H, Kumar K (2019) Linguistic interval-valued Atanassov intuitionistic fuzzy sets and their applications to group decision-making problems. IEEE Trans Fuzzy Syst. https://doi.org/10.1109/tfuzz.2019.2897961

Genest C, Mackay RJ (1986) Copulas Archimediennes et familles de lois bidimensionnelles dont les marges sont donn'ees. Can J Stat 14:145-159

Gou X et al (2018) Multiple criteria decision making based on distance and similarity measures under double hierarchy hesitant fuzzy linguistic environment. Comput Ind Eng 126:516-530

Herrera F, Herrera-Viedma E (2000) Linguistic decision analysis: steps for solving decision problems under linguistic information. Fuzzy Sets Syst 115:67-82

Herrera F, Martinez L (2000) A 2-tuple fuzzy linguistic representation model for computing with words. IEEE Trans Fuzzy Syst 8:746-752

Herrera F, Martinez L (2001) A model based on linguistic 2-tuples for dealing with multigranular hierarchical linguistic contexts in multi-expert decision-making. IEEE Trans Syst Man Cybern B Cybern 31:227-234

Jin FF et al (2019) A decision support model for group decision making with intuitionistic fuzzy linguistic preferences relations. Neural Comput Appl 31:1103-1124

Kaur G, Garg H (2018a) Cubic intuitionistic fuzzy aggregation operators. Int J Uncertain Quant. https://doi. org/10.1615/Int.J.UncertaintyQuantification.2018020471

Kaur G, Garg H (2018b) Multi-attribute decision-making based on Bonferroni mean operators under cubic intuitionistic fuzzy set environment. Entropy. https://doi.org/10.3390/e20010065

Kaur G, Garg H (2019) Generalized cubic intuitionistic fuzzy aggregation operators using t-norm operations and their applications to group decision-making process. Arab J Sci Eng 44:2775-2794

Khan MSA (2019) The Pythagorean fuzzy Einstein Choquet integral operators and their application in group decision making. Comput Appl Math 38:3. https://doi.org/10.1007/s40314-019-0871-z

Li CC, Dong Y, Herrera F et al (2017) Personalized individual semantics in computing with words for supporting linguistic group decision making An application on consensus reaching. Inf Fusion 33:29-40

Liu P, Liu X (2017) Multiattribute group decision making methods based on linguistic intuitionistic fuzzy power bonferroni mean operators. Complexity. https://doi.org/10.1155/2017/3571459 
Liu P, Qin X (2017) Maclaurin symmetric mean operators of linguistic intuitionistic fuzzy numbers and their application to multiple-attribute decision-making. J Exp Theor Artif Intell 29:1173-1202

Liu P, Wang P (2017) Some improved linguistic intuitionistic fuzzy aggregation operators and their applications to multiple-attribute decision making. Int J Inf Technol Decis Mak 16:817-850

Liu Y et al (2018) A multiple attribute decision making approach based on new similarity measures of intervalvalued hesitant fuzzy sets. Int J Comput Intell Syst 11:15-32

Liu Y et al (2019a) Dynamic intuitionistic fuzzy multiattribute decision making based on evidential reasoning and MDIFWG operator. J Intell Fuzzy Syst 36:5973-5987

Liu Y et al (2019b) A Novel Method Based on Extended Uncertain 2-tuple Linguistic Muirhead Mean Operators to MAGDM under uncertain 2-tuple linguistic environment. Int J Comput Intell Syst 12:498-512

Liu Y et al (2019c) Multiattribute group decision-making approach with linguistic pythagorean fuzzy information. IEEE Access 7:143412-143430

Liu Y et al (2019d) Pythagorean fuzzy linguistic muirhead mean operators and their applications to multiattribute decision making. https://doi.org/10.1002/int.22212

Lu X, Ye J (2019) Similarity measures of linguistic cubic hesitant variables for multiple attribute group decision-making. Information. https://doi.org/10.3390/info10050168

Mahmood T, Mehmood F, Khan Q (2016) Cubic hesistant fuzzy sets and their applications to multi criteria decision making. Int J Algebra Stat 5:19-51

Meng F et al (2019) Linguistic intuitionistic fuzzy preference relations and their application to multi-criteria decision making. Inf Fusion 46:77-90

Mishra AR et al (2019) Intuitionistic fuzzy divergence measure-based multi-criteria decision-making method. Neural Comput Appl 31:2279-2294

Mohd W, Rosanisah W, Abdullah L (2017) Aggregation methods in group decision making: a decade survey. Informatica 41(1):71-86

Muneeza AS (2020) Multicriteria group decision-making for supplier selection based on intuitionistic cubic fuzzy aggregation operators. Int J Fuzzy Syst. https://doi.org/10.1007/s40815-019-00768-x

Nelsen RB (1998) An introduction to copula. Springer, Berlin

Qiyas M, Abdullah S (2020) A novel approach of linguistic intuitionistic cubic hesitant variables and their application in decision making. Granul Comput. https://doi.org/10.1007/s41066-020-00225-3

Qiyas M, Abdullah S, Liu Y et al (2020) Multi-criteria decision support systems based on linguistic intuitionistic cubic fuzzy aggregation operators. J Ambient Intell Humaniz Comput. https://doi.org/10.1007/s12652020-02563-1

Rodriguez RM, Martinez L, Herrera F (2012) Hesitant fuzzy linguistic term sets for decision making. IEEE Trans Fuzzy Syst 20:109-119

Singh P (2015) Distance and similarity measures for multiple-attribute decision making with dual hesitant fuzzy sets. Comput Appl Math 36(1):111-126. https://doi.org/10.1007/s40314-015-0219-2

Szmidt E, Kacprzyk J (2000) Distances between intuitionistic fuzzy sets. Fuzzy Sets Syst 114:505-518

Tao $\mathrm{Z}$ et al (2018a) On intuitionistic fuzzy copula aggregation operators in multiple-attribute decision making. Cogn Comput 10:610-624

Tao $\mathrm{Z}$ et al (2018b) The novel computational model of unbalanced linguistic variables based on Archimedean Copula. Inte J Uncertain Fuzziness Knowl Based Syst 26:601-631

Torra V (2010) Hesitant fuzzy sets. Int J Intell Syst 25:529-539

Verma R (2014) A new measure of inaccuracy with its application to multi-criteria decision making under intuitionistic fuzzy environment. J Intell Fuzzy Syst 27(4):1811-1824

Verma R (2016) Multiple attribute group decision making based on generalized trapezoid fuzzy linguistic prioritized weighted average operator. Int J Mach Learn Cybern 8:1993-2007

Verma R (2020) On aggregation operators for linguistic trapezoidal fuzzy intuitionistic fuzzy sets and their application to multiple attribute group decision-making. J Intell Fuzzy Syst 38:2907-2950

Verma R, Merigó JM (2020) Multiple attribute group decision making based on 2-dimension linguistic intuitionistic fuzzy aggregation operators. Soft Comput 24:17377-17400

Verma R, Sharma BD (2013) Exponential entropy on intuitionistic fuzzy sets. Kybernetika 49(1):111-127

Wang JQ, Han ZQ, Zhang HY (2014) Multi-criteria group decision-making method based on intuitionistic interval fuzzy information. Group Decis Negot 23:715-733

Xu Z (2004) A method based on linguistic aggregation operators for group decision making under linguistic preference relations. Inform Sci 166:19-30

Xu ZS (2005) An overview of methods for determining owa weights. Int J Intell Syst 20:843-865

Ye J (2018) Multiple attribute decision-making method based on linguistic cubic variables. J Intell Fuzzy Syst 34:2351-2361

Zadeh LA (1965) Fuzzy sets. Inf Control 8:338-353 
Zadeh LA (1975) The concept of a linguistic variable and its application to approximate reasoning: Part-1. Inform Sci 8:199-251

Zhang H (2014) Linguistic intuitionistic fuzzy sets and application in MAGDM. J Appl Math. https://doi.org/ $10.1155 / 2014 / 432092$

Zhu B, Xu ZS, Xia MM (2012) Dual hesitant fuzzy sets. J Appl Math. https://doi.org/10.1155/2012/879629

Publisher's Note Springer Nature remains neutral with regard to jurisdictional claims in published maps and institutional affiliations.

\section{Affiliations}

\section{Hao bin Liu ${ }^{1,2,3} \cdot$ Yi Liu $^{1,2,3}$ (D) Lei Xu ${ }^{1,2,3} \cdot$ Saleem Abdullah ${ }^{4}$}

Hao bin Liu

njtcliuhb@163.com

Lei Xu

10001265@njtc.edu.cn

Saleem Abdullah

saleemabdullah@awkum.edu.pk

1 Data Recovery Key Lab of Sichuan Province, Neijiang Normal University, Neijiang 641000, Sichuan, People's Republic of China

2 School of Mathematics and Information Science, Neijiang Normal University, Neijiang 641000, Sichuan, People's Republic of China

3 Numerical Simulation Key Laboratory of Sichuan Province, Neijiang Normal University, Neijiang 641110, Sichuan, People's Republic of China

4 Department of Mathematics, Abdul Wali Khan University, Garden Campus, Mardan, Pakistan 